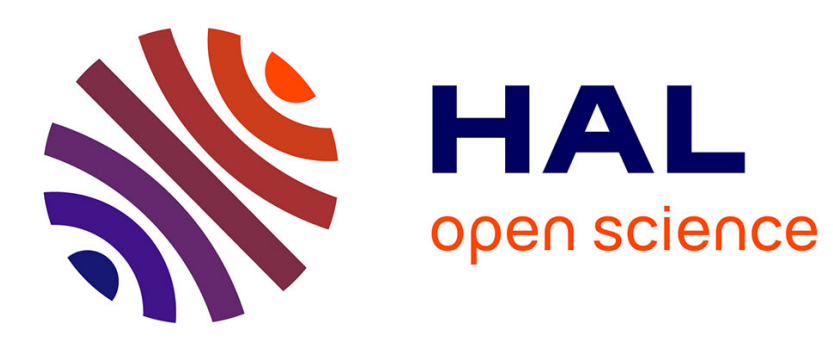

\title{
A systematic hybrid method for real-time prediction of system conditions in natural gas pipeline networks
} Huai Su, Enrico Zio, Jinjun Zhang, Zhe Yang, Xueyi Li, Zongjie Zhang

\section{To cite this version:}

Huai Su, Enrico Zio, Jinjun Zhang, Zhe Yang, Xueyi Li, et al.. A systematic hybrid method for real-time prediction of system conditions in natural gas pipeline networks. Journal of Natural Gas Science and Engineering, 2018, 57, pp.31-44. 10.1016/j.jngse.2018.06.033 . hal-01988963

\section{HAL Id: hal-01988963 https://hal.science/hal-01988963}

Submitted on 8 Feb 2019

HAL is a multi-disciplinary open access archive for the deposit and dissemination of scientific research documents, whether they are published or not. The documents may come from teaching and research institutions in France or abroad, or from public or private research centers.
L'archive ouverte pluridisciplinaire HAL, est destinée au dépôt et à la diffusion de documents scientifiques de niveau recherche, publiés ou non, émanant des établissements d'enseignement et de recherche français ou étrangers, des laboratoires publics ou privés. 


\title{
A systematic hybrid method for real-time prediction of system conditions in natural gas pipeline networks
}

\author{
Huai Su ${ }^{\mathrm{a}}$, Enrico Zio ${ }^{\mathrm{b}, \mathrm{c}}$, Jinjun Zhang ${ }^{\mathrm{a}, *}$, Zhe Yang ${ }^{\mathrm{b}}$, Xueyi Li $^{\mathrm{a}}$, Zongjie Zhang ${ }^{\mathrm{a}, \mathrm{d}}$ \\ ${ }^{a}$ National Engineering Laboratory for Pipeline Safety, MOE Key Laboratory of Petroleum Engineering, Beijing Key Laboratory of Urban Oil and Gas Distribution \\ Technology, China University of Petroleum-Beijing, 102249, Beijing, China \\ b Dipartimento di Energia, Politecnico di Milano, Via La Masa 34, 20156, Milano, Italy \\ ${ }^{\mathrm{c}}$ Chair System Science and the Energy Challenge, Fondation Electricité de France (EDF), CentraleSupélec, Université Paris Saclay, Bâtiment Bouygues, Laboratoire LGI, \\ 2ème étage, 3 rue Joliot-Curie, 91192, Gif-sur-Yvette cedex, France \\ d Petrochina West East Gas Pipeline, Dongfushan Road 458, Pudong District, 200122, Shanghai, China
}

\section{A R T I C L E I N F O}

\section{Keywords:}

Natural gas pipeline system

Deep learning

Data driven

Structural controllability theory

Real-time prediction

\begin{abstract}
A B S T R A C T
The current framework of management of natural gas pipeline systems, based on off-line simulation, is facing challenges because of the increasing complexity, uncertainty and a number of time-dependent factors. To be effective, it requires comprehensive knowledge of system characteristics, accurate initial and boundary conditions. In an attempt to circumvent these problems, in this work we propose to use the deep learning method in the natural gas transmission system operation and management context. A data-driven prediction method is developed from real-time data of operation pressure and gas consumption. Specifically, the deep learning method is combined with the data window method and structural controllability theory to predict the conditions of gas pipeline network components. The data window method is applied to reconstruct the data structure and build a "memory" for the deep learning method. Structural controllability theory is applied to extract critical parameters, for reducing the problem size. The developed method allows accurate and efficient predictions, especially in abnormal conditions. For demonstration, the method is applied to a complex gas pipeline network. The results show that the developed method can provide accurate real-time predictions useful for reducing potential losses in operation, and perform efficient and effective management of the gas pipeline system. In the case study, the average prediction accuracy is higher than 0.99 .
\end{abstract}

\section{Introduction}

Natural gas travels a long distance, from sources through pipeline networks to the different kinds of customers. Accurate and timely operations ensure reliable supply to the customers. This requires timely precise knowledges of conditions of the system and of its components (Chertkov et al., 2015). Hence, it is crucial to use efficient and effective methods for accurately predicting the system dynamic responses and the components future conditions.

Off-line simulation is typically applied for the analysis, decision support and optimization of pipeline networks (Fasihizadeh et al., 2014; Zhang et al., 2016b). Many efforts have been made for the improvement of the numerical models (Wang et al., 2015; Pambour et al., 2016a) and model solvers (Wang et al., 2018; Behbahani-Nejad and Bagheri, 2010). Some unconventional off-line simulation methods have also been developed (Madoliat et al., 2016; Uilhoorn, 2017). However, an accurate simulation requires exact conditions, e.g., comprehensive system characteristics, accurate initial states and imposed boundary conditions. These are unfortunately difficult to obtain in practice. Furthermore, off-line simulation has difficulties in accounting for the time-dependent factors of the system dynamics and in treating the uncertainties in the model and its parameters. Finally, the computational burden can be quite significant for complex pipeline networks.

Form a different context, we observe that the innovations brought by artificial intelligence, machine learning and big data are changing the vision of traditional energy industry. Many researches have been carried out attempting to solve various challenges which energy systems are currently facing, e.g., system reliability and stability (Zio and Di Maio, 2010; Fang and Zio, 2013; Zhang et al., 2016a), operation efficiency and cost control (Hegde and Gray, 2017; Azadeh et al., 2016), renewable energy management (Lou et al., 2016) and environment issues (Tan et al., 2016). The application of techniques of forecasting (Wang et al., 2016; Kalantari-Dahaghi et al., 2015), classification (Hu et al., 2010; Pooyan et al., 2015) and optimization (Azadeh

\footnotetext{
* Corresponding author. College of Mechanical and Transportation Engineering, China University of Petroleum, Fuxue Road 18, Changping District, 102249, Beijing, China.

E-mail address: zhangjj@cup.edu.cn (J. Zhang).
} 
et al., 2016; Xiong et al., 2018) has been successfully explored in different energy systems, to the benefit of regulators, customers and operators. Besides, recurrent and cascade neural networks are among the best choices for dynamic system predictive modeling (Vaferi et al., 2015; Lashkarbolooki et al., 2013; Güler and Übeyli, 2006; Güler et al., 2005).

In natural gas pipeline network systems, large amounts and various types of data of operation, device status and gas consumption are generated and collected by SCADA (Supervisory Control and Data Acquisition) systems. Based on these (big) data, some efforts have been made for online dynamic state estimation and forecast of gas demand. The online state estimators are mostly developed based on filter models (Durgut and Leblebicioğlu, 2016), which are applied to estimate the real time state of pipeline networks. Applications of machine learning for forecasting natural gas demands have drawn great attention from both research and practice perspectives (Panapakidis and Dagoumas, 2017; Yu and Xu, 2014). Many algorithms have been developed to predict natural gas demand over different time horizons.

In natural gas pipeline networks, the future states of the components and the system dynamics depend on the previous state history and external disturbances. Because of the complex system structure of the pipeline network and the complex transient process of gas flow in the pipelines, traditional machine learning methods have difficulties in accurately regressing the dynamic behaviors of complex gas pipeline networks.

Recently, deep learning, a type of machine learning algorithm, has drawn a lot of interest from industry and academics. Deep learning has already been successfully used in object detection (Kong et al., 2018; Hu et al., 2017), dimensionality reduction (Turati et al., 2017), natural language processing (Evermann et al., 2017) and other applications. Deep architectures, or multiple layer architectures, are used to extract features in data layer by layer, and the inherent features of the dynamics in a given pipeline system can be found from the data (Hinton et al. 2006). Indeed, the complicated dynamic behaviors of a gas pipeline network can be "learned" by deep learning without prior knowledge, and the system dynamics can be accurately predicted. Although deep learning has been applied in the area of natural gas pipeline modeling and analysis, most of the works focus on the analysis of single units (such as compressor stations) or pipelines (Qiu et al., 2015). The application for the analysis of the dynamics of a complex pipeline network needs to be further explored.

In this paper, we develop a deep learning-based method to predict system dynamic behavior and component states in large, complex natural gas pipeline networks. A stacked-auto-encoder model is trained in the layerwise greedy fashion and applied for learning the system dynamic features. The correlations of temporal and spatial factors are inherently considered in the model. Network structural controllability theory and the data-window method are integrated with the deep learning method, for a more efficient use of the data.

The rest of the paper is organized as follows: Section 2 introduces the development of the deep learning prediction model and the method to train it; the data-window method is introduced in Section 2.4. The data selection method, based on network structural controllability theory, is introduced in Section 3. In Section 4.1, the accuracy of the developed method is verified by benchmarking against shallow neural networks and support vector machine (SVM), for a triangle pipeline network; in Section 4.2, the deep learning model is applied to a complex natural gas pipeline network in both normal and abnormal conditions, and the results are discussed in detail.

The main contributions of this work include the following:

1 A method for natural gas pipeline dynamic behavior prediction is developed. The deep learning method shows a stable performance under different conditions and is able to provide effective information for decision support. It can accurately predict the system responses under abnormal conditions without prior knowledge, which can help to improve the efficiencies of preventive actions and to reduce potential losses.

2 To some extent, this work paves the way for the application of deep learning to complex gas transmission systems. Indeed, this work shows that deep learning is very powerful in learning the complex dynamic features of gas pipeline networks, which is crucial for demand-side management, detection and early-warning, decision support and so on.

\section{The deep learning prediction method}

Deep learning has multiple processing layers, which allow learning features of data without prior knowledge (Hinton et al. 2006). In this section, inspired by the work performed by (Lv et al., 2015), a stacked auto-encoder (SAE) model is applied for learning generic features of condition data in gas pipeline networks. A regression layer is stacked on the top of the SAE to perform the prediction based on the learned features. For completeness of the paper, the underlying principle of the $\mathrm{SAE}$ and of the training process are recalled in what follows.

\subsection{Auto-encoder}

An auto-encoder is a neural network that attempts to reconstruct its input at the output layer, after passing through intermediate, hidden layers. A sample auto-encoder model, which has one input layer, one output layer and a hidden layer, is presented in Fig. 1. Given a training sample, $\boldsymbol{x}=\left\{x_{1}, x_{2}, \ldots, x_{n}\right\}$, an input $x_{1}$ is firstly encoded by an autoencoder model to a hidden representation $y\left(x_{1}\right)$ based on Equation (1), and then $y\left(x_{1}\right)$ is decoded back as $z\left(x_{1}\right)$ by Equation (2):

$\mathbf{y}(\mathbf{x})=f\left(\mathbf{W}_{1} \mathbf{x}+\mathbf{b}\right)$

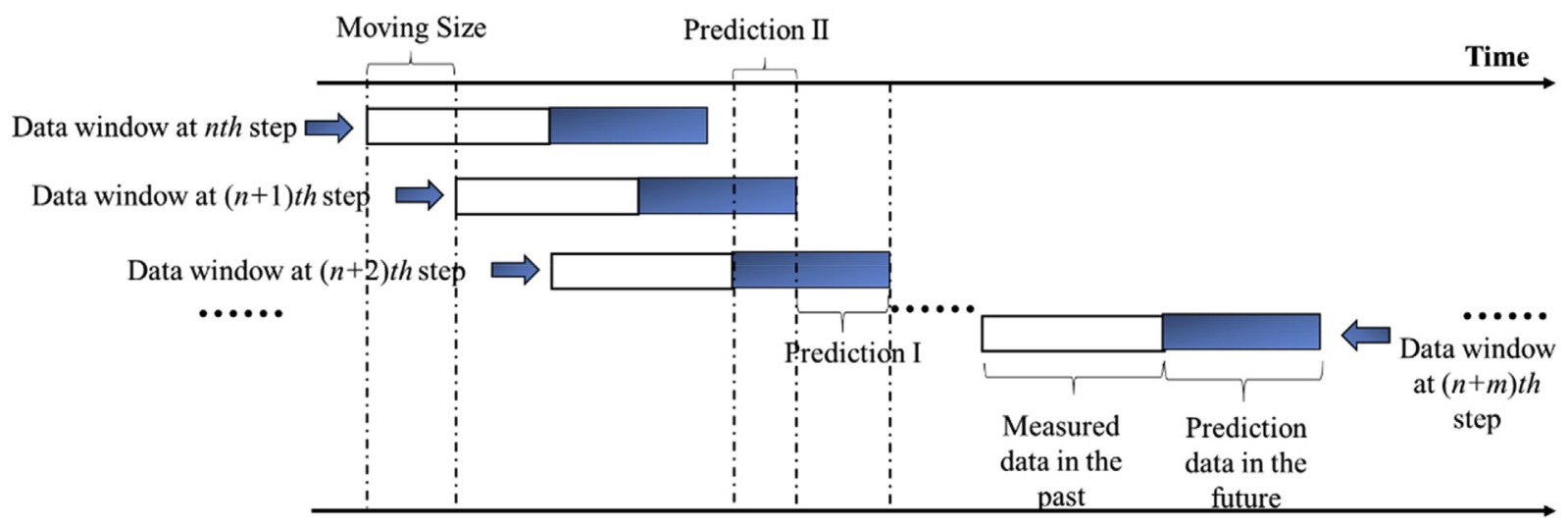

Fig. 1. A sample auto-encoder. 
$\mathbf{z}(x)=g\left(\mathbf{W}_{2} \mathbf{y}(x)+\mathbf{c}\right)$

where $\mathbf{W}_{1}$ represents the weight matrix; $\mathbf{b}$ represents the vector of encoding bias; $\mathbf{W}_{2}$ is the decoding matrix; $\mathbf{c}$ is the vector of decoding bias. In this paper, "logic sigmoid" functions of the type in Equation (3) are applied for $f$ and $g$ :

$f(x)=\frac{1}{1+e^{-x}}$

By minimizing the error of reconstruction $(E)$, the model parameters $\theta$ can be obtained:

$\theta=\arg _{\theta} \min E(\mathbf{x}, \mathbf{z})=\arg _{\theta} \min \frac{1}{2} \sum_{i=1}^{n}\left\|x_{i}-z\left(x_{i}\right)\right\|^{2}$

Sparsity constraints are supplemented into the objective function, considering that if the size of the input layer is no larger than that of the hidden layer, the above method may potentially learn the identify function. By this way, the auto-encoder becomes a spare auto-encoder, and the reconstruction error minimization problem is transformed to:

$S=E(\mathbf{x}, \mathbf{z})+\phi \sum_{j=1}^{H} K L\left(\rho \| \hat{\rho}_{j}\right)$

where $H$ is the number of hidden units; $\phi$ represents the weight of the sparsity term; $\rho$ represents the sparsity parameter, which is usually a small value (close to 0$) ; K L\left(\rho \| \hat{\rho}_{j}\right)$ is Kullback-Leibler (KL) divergence (in Equation (7)); $\hat{\rho}_{j}$ is the average activation of the hidden unit $j$ (in Equation (8)).

$K L\left(\rho \| \hat{\rho}_{j}\right)=\rho \log \frac{\rho}{\hat{\rho}_{j}}+(1-\rho) \log \frac{1-\rho}{1-\hat{\rho}_{j}}$

$\hat{\rho}_{j}=\frac{1}{N} \sum_{i=1}^{n} y_{j}\left(x_{i}\right)$

\subsection{Stacked auto-encoders}

Recent works of machine learning have shown the great power of a deep architecture to learn highly non-linear and complicated patterns in data (Qiu et al., 2015). Inspired by these works, a SAE is developed in this work, made of several auto-encoders stacked one on top of another, in which the input of the upper layer is taken from the output of the lower hidden layer. The SAE has a significant advantage for finding the highly non-linear patterns between the collected data (current and historical) and to extract the features of the dynamics (Lv et al., 2015).

To use the SAE model for condition prediction in natural gas pipeline networks, a standard predictor is stacked on the top layer. In this paper, a logistic regression layer is applied for supervised system condition prediction. The predictor and the SAE model comprise the whole deep learning model for condition prediction in natural gas pipeline networks, as illustrated in Fig. 2:

\subsection{Training process and algorithm}

BP method can be directly used to train conventional neural networks, by gradient-based optimization. Unfortunately, deep neural networks trained by BP method have bad performances. On the contrary, the greedy layerwise unsupervised algorithms have been developed with successful results (Bengio et al., 2007). The key points are: firstly, the greedy layerwise unsupervised algorithm is used to pre-train the deep network layer by layer, from the bottom to the top; then finetuning based on BP is applied to tune the parameters in the model in a top-down way, to improve performance (Hinton et al., 2006). The training procedure is shown in Fig. 3.

We notice that the deep learning model needs to be adjusted and retrained with new data on a regular basis, to ensure the model is capable to maintain a good performance under a changing environment.

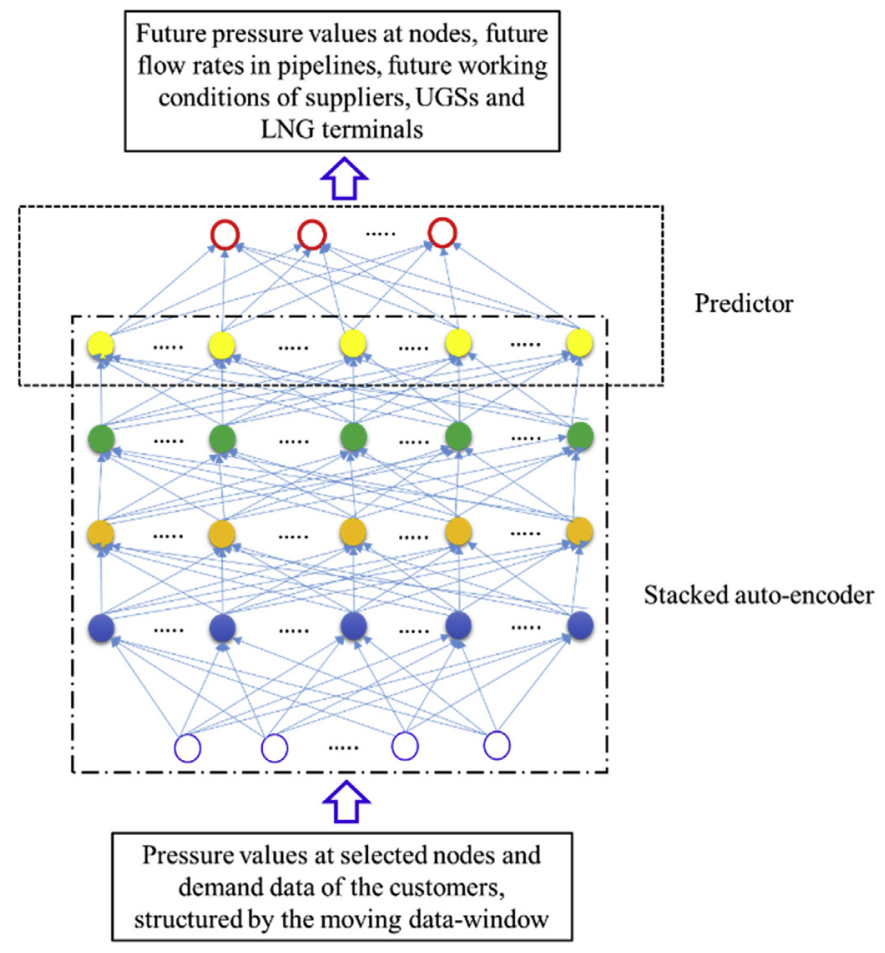

Fig. 2. Illustration of the deep learning model for on-line condition prediction for natural gas pipeline networks.

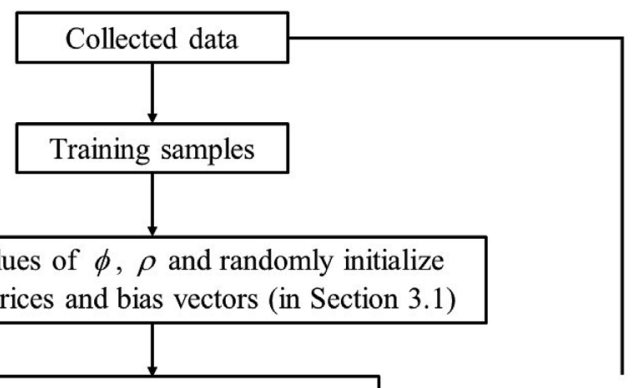

Unsupervised training of hidden

layers via the greedy layer-wise algorithm

Set the values of $\phi, \rho$ and randomly initialize weight matrices and bias vectors (in Section 3.1)

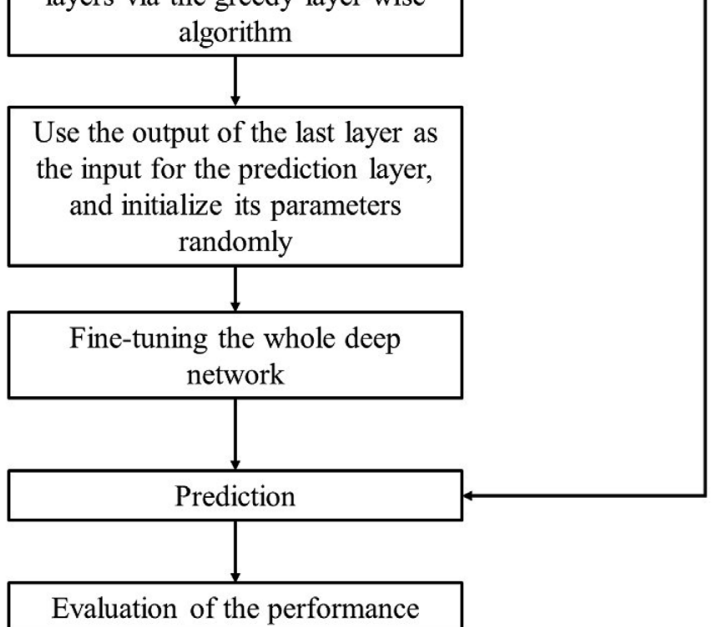

Fig. 3. The flowchart of the training process.

\subsection{Moving data window method and model updating}

For a short-term condition prediction, the future evolution is 


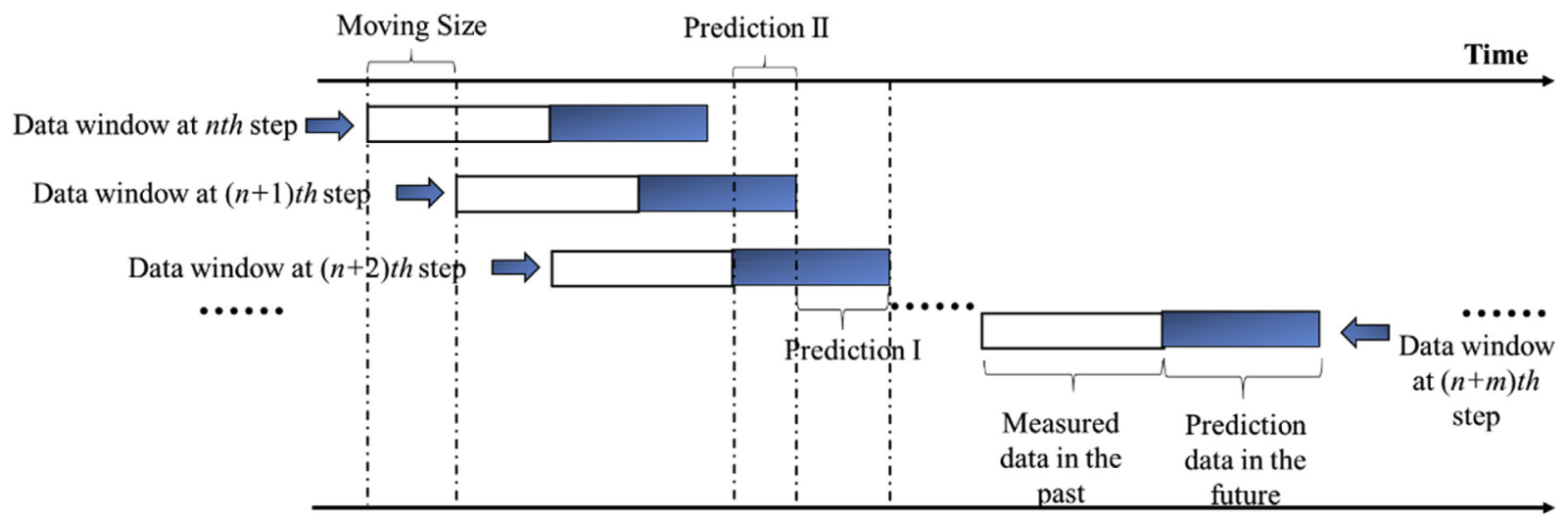

Fig. 4. The schematic illustration of the moving data window method.

determined by the conditions of the recent past (depending on the scale of the network system). Given that, we need to reconstruct the historical data for the prediction model in order to create a "memory" of the effective data. Hence, in this paper, we apply a data-window method (Zhou et al., 2017), which is illustrated in Fig. 4.

In Fig. 4, the length of a moving data window is made of two parts: input (white region: data measured in the past) and prediction (dark region: data predicted in the future). At every prediction step, the data in the white region are used as input of the deep learning model and the data in the dark region are the predicted future data. At each prediction step, the overlapped previous prediction data (Prediction I) is replaced by the new prediction results obtained (Prediction II), for improved prediction performance.

A proper design of the length of the moving data window can contribute to a good prediction performance, with lower computational storage cost. Actually, the length of the white region works as the "memory capacity" of the deep learning model. Hence, a well-designed data window helps the deep learning model efficiently remember most relevant data for prediction. In this paper, for convenience of illustration, we assume that the future condition of the natural gas pipeline network is determined by the past system conditions (the internal property) and the fluctuations of gas demands (the external influence), based on which a criterion for the design of the input windows is developed as Equation (8):

$S=\max \left\{T, \max \left\{P_{1}, P_{2}, \cdots, P_{n}\right\}\right\}$

where $S$ is the designed length of the input part; $T$ is the longest time that a disturbance takes to spread to the whole pipeline network system; $P_{i}$ is the shortest period that can influence the future demand evolution of gas demand $i$. According to the works for demand prediction, the demands of gas can be influenced by various of factors (such as weather, demand history, population and so on) (Karadede et al., 2017). In this work, without lack of generality, we only consider demand history.

For the prediction part, the length of window is a trade-off between accuracy and efficiency. A longer prediction contributes more to the operation and management, but the accuracy of prediction will degenerate due to the increasing uncertainties. Theoretically, the length of prediction window should be within the value $(S)$ calculated by Equation (8), because of the prediction mechanism of the developed method. However, in practice, it should be designed based on the needs of the real world applications and the experience of the analysts. In general, the deep learning model is pre-trained and the related collected real-time data are organized by the data window as the input. The deep learning model will be updated by new data collected in a specific time interval. In the future, we will develop the deep learning model in sequential mode, which can further improve the ability of the model.

\section{Data preprocessing for input to the deep learning model}

Direct use of the sensor collected data may lead to high storage burden and degenerated prediction (Xue et al., 2017). Valuable data must be selected from the collected data, to reduce the problem size and convert the "big data" to "wise data".

The dynamic evolution of the gas pipeline system is driven by pressure (Pambour et al., 2016b) and the control theory enables that the system be fully controlled. Then, valuable data are the pressures of these elements in the network which are most important for system controllability, i.e. we can attempt to use the pressure data (historical and current) of these elements to predict the condition of the overall pipeline network.

In this paper, network structural controllability theory (Leitold et al., 2017) is applied to identify the elements which can control the overall gas pipeline network. This method has been used in real-world complex network systems including gas pipeline networks (Han et al., 2015). The results of the applications show the capability of effectively identifying the driver nodes for the system structural control.

Generally, the natural gas pipeline network is driven by nonlinear processes, but in many aspects, the controllability of nonlinear systems is structurally similar to that of linear systems (Chiang and Zavala, 2016). Then, the canonical linear and time-invariant dynamics can be used to identify the driver nodes:

$\frac{d \mathbf{x}(t)}{d t}=\mathbf{A x}(t)+\mathbf{B u}(t)$

where the vector $\mathbf{x}(t)$ represents the states of the $N$ nodes in the natural gas pipeline network system at time $t$; the $N \times N$ matrix A describes the interaction strengths between nodes on the connected structure of the pipeline network; B is an input matrix identifying the driver nodes controlled by the time dependent input vector $\mathbf{u}(t)$.

In classical dynamic control theory, Kalman's rank condition can be used as criterion of controllability and to identify the minimum number of driver nodes (Kalman, 1963). However, the classical concept has difficulties when dealing with complex pipeline networks and new concepts of complexity are needed (Zio, 2016; Han et al., 2015): (1) the classical method is usually applied to undirected networks, but natural gas pipeline networks are directed; (2) to apply the Kalman's rank condition, we need to know the weights of all links (i.e. the elements in matrix A), which are usually unknown. (3) even if the weights can be approximated, we need a brute-force search to compute the rank for $2^{N}$ 1 combinations, which is impossible for large pipeline networks.

To bypass the problem, the analytical methods in (Liu et al., 2011) is applied here to identify the minimum set of the driver nodes. The socalled structural controllability of a system (A, B) allows determining the free parameters in $\mathbf{A}$ and $\mathbf{B}$. A and $\mathbf{B}$ are structured matrices whose elements are independent free parameters or zeros. In general, a structural controllable network system is controllable for most of 

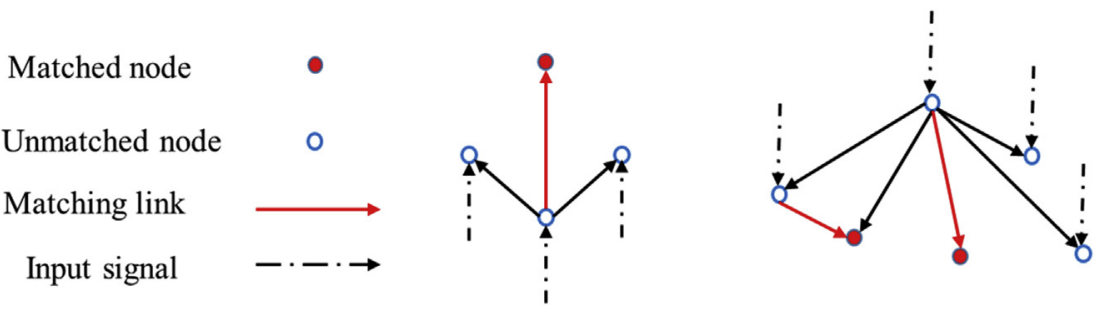

Fig. 5. Structural controllability of simple networks.

weight combinations, except for several pathological cases with zero measures (Shields and Pearson, 1976). Therefore, structural controllability theory can help to solve the inherently incomplete information of the elements in matrix $\mathbf{A}$.

Based on the concepts of structural controllability theory, the matching concept in graph theory (Leitold et al., 2017) is applied here for finding the minimum set of driver nodes. A matching set is a set of arcs without common vertices, in a directed graph G. A matched node is an ending vertex of an arc in the matching; otherwise, the node is unmatched (as shown in Fig. 5). The pipeline network can be fully controlled if and only if all unmatched nodes are directly controlled and the input signals can be transmitted to all matched nodes (Leitold et al., 2017). Hence, we need to find a maximum match (maximum matching may be not unique in a given network), which corresponds to a minimum set of driver nodes, which represents a minimum but effective set for prediction. In a given directed graph, the maximum matching can be determined in at most $O\left(N^{1 / 2} L\right)$ steps, where $L$ represents the number of arcs.

According to the inherent concept of structural controllability and the properties of the gas transportation process, the evolution of the overall gas pipeline network is determined by the pressure changes of the driver nodes. Hence, we can use the current and historical pressure data of these driver nodes to predict the system evolution. For a complex pipeline network, this method is capable to significantly reduce the problem size, while still achieving a good prediction performance.

\section{Application}

The deep learning model is applied to a simple triangle pipeline network and a part of a real-world pipeline network. The fluctuations in the natural gas market are not completely random and are driven by nonlinear dynamics, e.g., chaos. In this application, the gas demand fluctuations are generated by Mackey-Glass model (Equation (10)), a periodic and chaotic time-series model which is typically used to test the performances of predictive models because of its chaotic behavior (Sharma et al., 2016):

$\frac{d x(t)}{d t}=\frac{a x\left(t-\tau_{M}\right)}{1+x^{c}\left(t-\tau_{M}\right)}-b x(t)$

where $\tau_{M}$ is the time delay parameter ( $>16.8$ ), which determines the chaotic behavior of the time series. In this case, the value of $\tau_{M}$ is set to be 20. The parameters i.e., $a, c$ and $b$ are constants: $a=0.2, b=0.1$, $c=10$. The 4th Runge-Kutta method is used here to generate the time series data and then the data is sampled at a given interval. In this chaotic times series, current data values are dependent on those of the past, which is similar with gas demands fluctuations. Further, a random term (of $1 \%$ of the nominal value of the generated gas demands) is introduced, to make the application more realistic.

The network "real-time" operation data are generated by TGNET. Developed by Energy Solution, TGNET is a commercial software for steady state and transient thermal-hydraulic simulation of gas pipeline networks (Faertes et al., 2010). This software has been widely used in many areas, such as, pipeline design, risk assessment, contingency planning and so on. The simulation is carried out based on the following principles and assumptions:
(1) The active components are set at specific control modes, with desired set values;

(2) In the normal scenario, the system condition changes along with the fluctuations of demands;

(3) System conditions data are collected at given time intervals (each $15 \mathrm{~min}$ in the case) by pressure sensors and flow rate sensors.

In practice, the situation is more complex but in this work we mainly focus on deep learning and its ability to predict the nonlinear evolution process of gas pipeline networks.

The size of the input layer of the deep learning model is determined by the input data, selected by the structural controllability and reconstructed according to the data-windows. The structure of the hidden layers (i.e. the number of hidden layers and the numbers of each nodes in each hidden layer) is founded based on the prediction performances, by "try and error" based on grid search (Lv et al., 2015). The system conditions which are predicted as output are the pressures of the network nodes, the average flow rates in the pipelines and the amount of gas supplied by the suppliers.

For effective learning, the range of input data are normalized within $[0,1]$. Considering the big differences between the data sets collected from different parts of the pipeline network, the normalizations are performed separately for each data set.

The parameters of the equations in Section 2.1 and of the training process are: the weight of the sparsity term is 0.75 ; the average activation is 0.6 ; sparsity parameter is 0.0004 .

To present the way to use the developed method, the flowchart of the whole process is showed in the following Fig. 6:

\subsection{Triangle pipeline network}

We consider the simple triangle pipeline network in Fig. 7 and benchmark the developed deep learning model against two conventional machine learning methods-support vector machine (SVM) and BP neural network. We choose the simple network so as to eliminate the inherent advantage of the deep learning model in processing big data. To prove the advantage of the deep learning model over the shallow neural network and the classical machine learning model, the shallow BP network only has one layer. But the parameters of the shallow BP network and the SVM model have been well designed by "trial and error", to make sure the results can represent their best abilities. Node 1 is the supplier and Nodes 2, 3 are the customers. The control mode of the supply node is pressure-controlled (maintaining constant pressure of $5 \mathrm{MPa}$ ) and the control mode of the customers is flow rate-controlled (the boundary conditions of the flow rates are shown in Fig. 8). The diameters of the three pipelines are $0.6 \mathrm{~m}$ and lengths of pipelines 1-3, $1-2$ and $2-3$ are $80 \mathrm{~km}, 90 \mathrm{~km}$ and $100 \mathrm{~km}$, respectively. The parameters and the algorithm of the $\mathrm{T}-\mathrm{H}$ simulation are chosen according to the default settings of the TGNET software.

In this case, the size of the input window is $10 \mathrm{~h}$ and sizes of the prediction window are selected as $3 \mathrm{~h}, 5 \mathrm{~h}$ and $9 \mathrm{~h}$, in order to test the prediction performances under different lengths of prediction time. For the simple network, we assume that the loads at the two demand sites and the pressure of Node 3 , which are used as the input of the prediction model, can be collected. Therefore the input size for the 


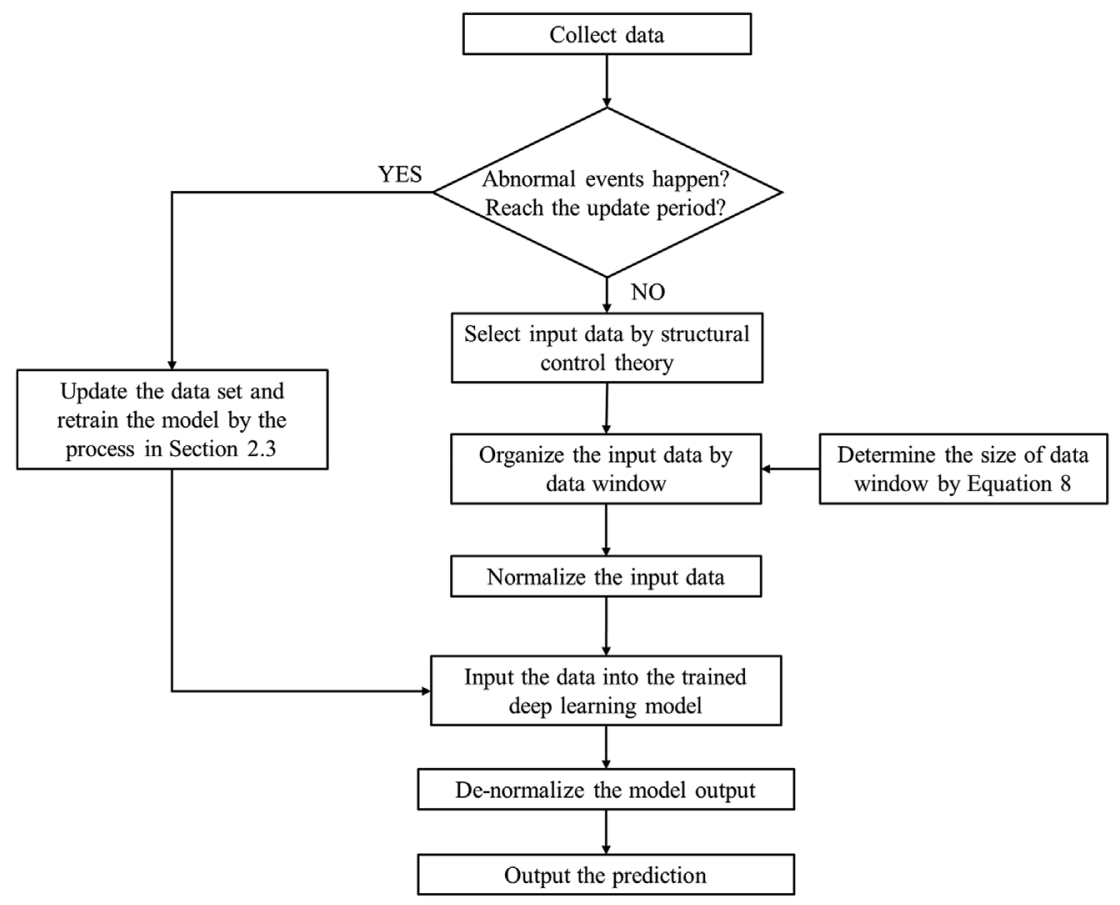

Fig. 6. The flowchart of the application process of the whole method.

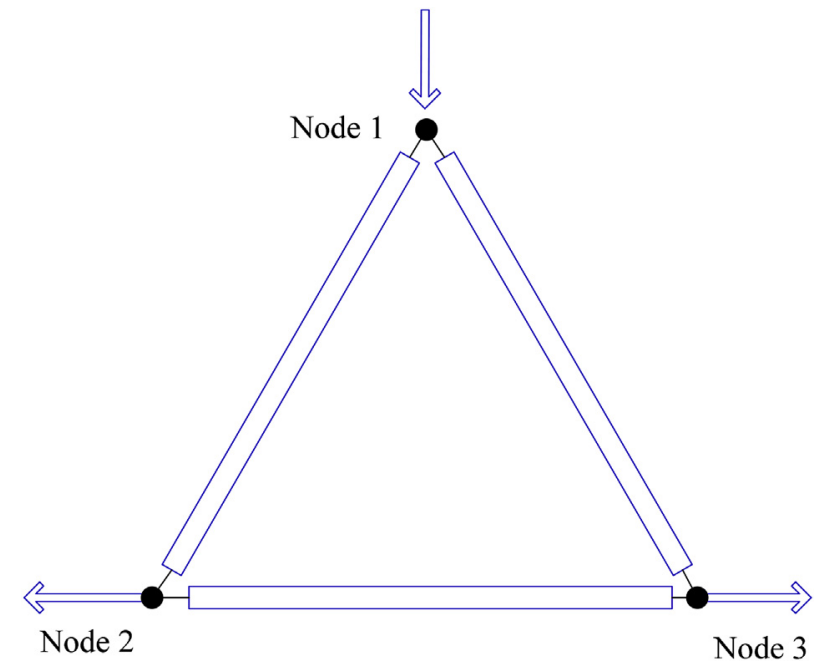

Fig. 7. Layout of the triangle gas pipeline network.

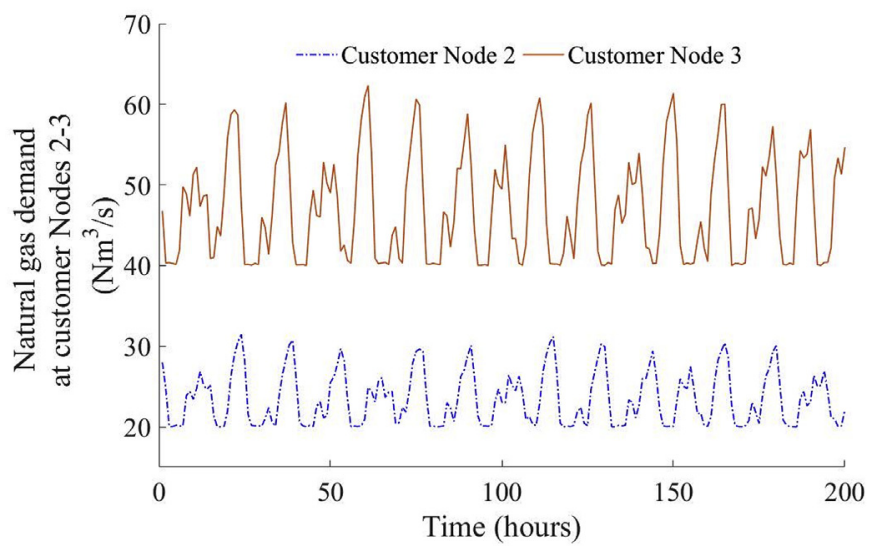

Fig. 8. The generated demands at customer Nodes $2-3(0-200 \mathrm{~h})$. prediction problem of the triangle network is 120 (the length of the input window is $10 \mathrm{~h}$ and the sampling frequency is every $15 \mathrm{~min}$ ). The $\mathrm{T}-\mathrm{H}$ dynamic simulation is carried out for $1000 \mathrm{~h}$ and the results are organized by the data window as data sets (with the size of 4900) for model training, adjusting and testing, following a "50\%-30\%-20\%" partition (50\% data for training the model, $30 \%$ for adjusting the model and $20 \%$ for testing the prediction performance). The model optimization and validation are performed based on different data sets, to make sure the model is generalized. The structures and settings of the deep learning model will change along with the length of prediction time. In the "trial and error" optimization, the sizes of the hidden layers are chosen from 2 to 6 , and range of the number of the units in the hidden layers is $[300,250,200,150,50]$. For the $3 \mathrm{~h}$ prediction, the best architecture is a two-layer with hidden units of $\{200,150\}$; for the $5 \mathrm{~h}$ prediction, the best architecture is a two-layer with hidden units of $\{250,100\}$; for the $10 \mathrm{~h}$ prediction, the best architecture is a three-layer with hidden units of $\{250,150,150\}$.

Portions of the generated demands at customer Nodes 2-3 are shown in Fig. 8, from which we can see that the generated chaotic demand data are to some extent periodical, but not completely cycling, like the changes of demands of gas in real world.

The prediction performance is measured by three indices: mean absolute error (MAE), root-mean-square error (RMSE) and mean relative error (MRE). In Tables 1 and 2, the performances of different methods are compared. Table 1 presents the results of pressure prediction of customer Node 2 and Table 2 presents the results of gas flow rate prediction at Supply node 1 . From the Tables, we see that the deep

\section{Table 1}

Prediction performance comparison of the deep learning model, the BP Neural Network and the SVM, based on the results of pressure prediction of customer Node $2\left(\times 10^{3} \mathrm{~Pa}\right)$.

\begin{tabular}{lllllll}
\hline Task & \multicolumn{2}{l}{ The deep learning model } & \multicolumn{2}{l}{ BP Neural Network } & \multicolumn{2}{l}{ SVM } \\
\cline { 2 - 7 } & MAE & RMSE & MAE & RMSE & MAE & RMSE \\
\hline 3 h prediction & 2.90 & 3.45 & 12.63 & 17.22 & 17.11 & 22.30 \\
5 h prediction & 3.92 & 4.69 & 16.99 & 21.72 & 19.39 & 22.98 \\
9h prediction & 4.58 & 5.67 & 25.78 & 27.23 & 27.18 & 31.10 \\
\hline
\end{tabular}


Table 2

Prediction performance comparison of the deep learning model, the BP Neural Network and the SVM, based on the results of gas flow rate prediction of supply Node $1\left(\mathrm{Nm}^{3} / \mathrm{s}\right)$.

\begin{tabular}{lllllll}
\hline Task & \multicolumn{2}{l}{ The deep learning model } & BP Neural Network & \multicolumn{2}{l}{ SVM } & \\
\cline { 2 - 7 } & MAE & RMSE & MAE & RMSE & MAE & RMSE \\
\hline 3 h prediction & 0.88 & 1.04 & 3.63 & 4.91 & 5.00 & 6.32 \\
5 h prediction & 1.15 & 1.37 & 5.06 & 6.43 & 5.73 & 6.75 \\
9h prediction & 1.31 & 1.43 & 6.00 & 6.32 & 7.94 & 9.71 \\
\hline
\end{tabular}

learning model is more accurate than the other competing methods, for the different lengths of prediction time. From Tables 1 and 2, we can observe that the accuracy decreases with increasing prediction time. Generally, this is because the strength of the relationship between the current condition and the future condition decreases as the prediction time horizon increases, and accordingly increases the difficulty for the neural networks to learn such relationship. To overcome this issue controlled recursive processes that make use of the predictions can enhance the ability of the deep learning model.

The visual displays of the results of MRE are given in Figs. 9-11 in terms of the Empirical CDF, which describes the variability of the predictions of the system conditions due to the stochasticity of the demands. Also these results show that the deep learning model outperforms the BP neural network and SVM. For the pressure prediction, the deep learning model has an accuracy of more than $99 \%$ at any length of time, and most of its flow rate predictions have an accuracy of more than $98 \%$.

\subsection{Complex gas pipeline network}

The deep learning model is applied to a relatively complex gas pipeline network, which comprises two pipeline importers, 37 pipelines (total length of approx. $1100 \mathrm{~km}$, diameters ranging from $950 \mathrm{~mm}$ to $1014 \mathrm{~mm}$ ), 23 demand sites (including city gates, factories, power plants and export stations), seven regulation stations, two compressor stations (pressure ratios ranging from 1.02 to 1.18), one LNG terminal and one UGS. The control modes of the two pipeline importers are pressure-controlled, while the LNG terminal and the UGS are set at flow rate-controlled modes. The boundary conditions of the flow rates at the demand nodes are generated by the model of Equation (10). The supply pressures provided by the two compressor stations are maintained at the set points of $7 \mathrm{MPa}$ (Importer 1) and 6.5 MPa (Importer 2), respectively. The regulation stations are set as inactive Modes. The gas pipeline network system is presented in Fig. 12. In the Figure, the customers are represented by the polygons and the nodes selected by structural controllability are at the nodes linked to Customer 6, Customer 13, Customer 10 and Customer 8.
The size of input data window is chosen to be $12 \mathrm{~h}$ and the input size is 1200 (including 21 customer demands and 4 selected nodes). The T-H dynamic simulation is carried out for $2000 \mathrm{~h}$ and the results are organized by the data window and used as data sets with the size of 7900 , for model training, adjusting and testing, following the same " $50 \%$ $30 \%-20 \%$ " principle. In the "trial and error" optimization for the condition of input of demands and selected nodes, the sizes of the hidden layers are chosen from 2 to 6 , and the range of the number of the units in the hidden layers is $[2000,1750,1500,1250,1000,750,500]$. For the $5 \mathrm{~h}$ prediction (the benchmark in this case), the best architecture is a three-layer with hidden units of $\{1750,1250,1250\}$. The prediction performances are measured by MAE, RMSE and MRE.

Firstly, to verify the effectiveness of the data selection method proposed in Section 3, comparisons are made of the predictions based on three different sets of input data:

A Pressure data of all nodes and demands data;

B Pressure data of only the nodes selected based on the structural controllability and demands data;

C Only demands data.

The MAE and RMSE results of the three prediction performances are presented in Tables 3 and 4, with reference to gas flow rate predictions and node pressure predictions. In these Tables, we can see that the predictions based on input sets A and B have similar accuracy, and definitely superior to that based on input set C. This is also shown by the empirical CDF of the relative error values shown in Fig. 13.

To test the capacity of the developed model on different time lengths of predictions, the predictions are performed for $2 \mathrm{~h}, 5 \mathrm{~h}, 8 \mathrm{~h}$, $10 \mathrm{~h}$ and $15 \mathrm{~h}$. The MAE and RMSE results are presented in Table 5 and the empirical CDFs of the relative error values are shown in Fig. 14. From these results, we can see that the deep learning model gives good performances for predictions upto $10 \mathrm{~h}$ and the values of MAE and RMSE in Table 5 double from $2 \mathrm{~h}$ to $15 \mathrm{~h}$. In Fig. 14, the gap between the empirical CDFs of $15 \mathrm{~h}$ and $10 \mathrm{~h}$ is larger than that of $5 \mathrm{~h}$ and $10 \mathrm{~h}$.

From Tables 4-6, we can observe large errors in the pressure predictions due to the normalizing and de-normalizing processes. However, the relative errors (presented in Figs. 12-14), which reflect the true prediction ability of the model, are maintained at a relatively low level $\left(10^{-4}-10^{-3}\right)$. Besides, an accuracy improvement can be observed by comparing the results in Section 4.2 and those in Section 4.1, which means that the prediction performance can be enhanced by increasing the size of data, even if the complexity of the network has significantly increased.

Generally, the data collected by sensors are noisy. The prediction model should, then, be capable of making accurate predictions with noisy data. Thus, five levels of artificial noises are introduced (i.e. \pm $0.5 \%, \pm 1.0 \%, \pm 1.5 \%, \pm 2.0 \%, \pm 2.5 \%$ of the nominal values of
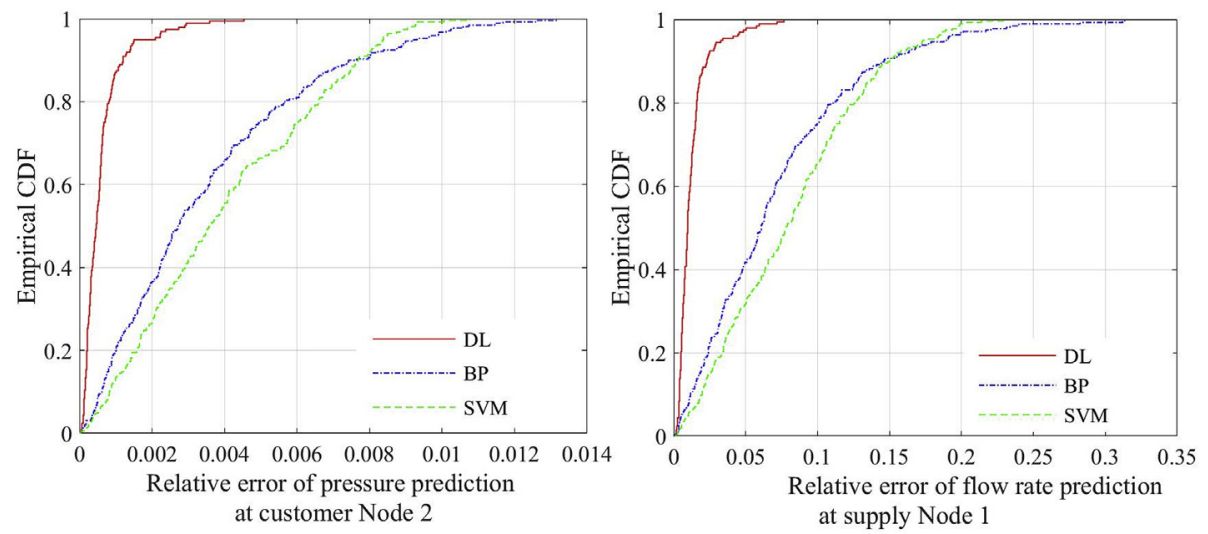

Fig. 9. Performance comparison based on the empirical CDF of the relative error of prediction results ( $3 \mathrm{~h}$ prediction). 

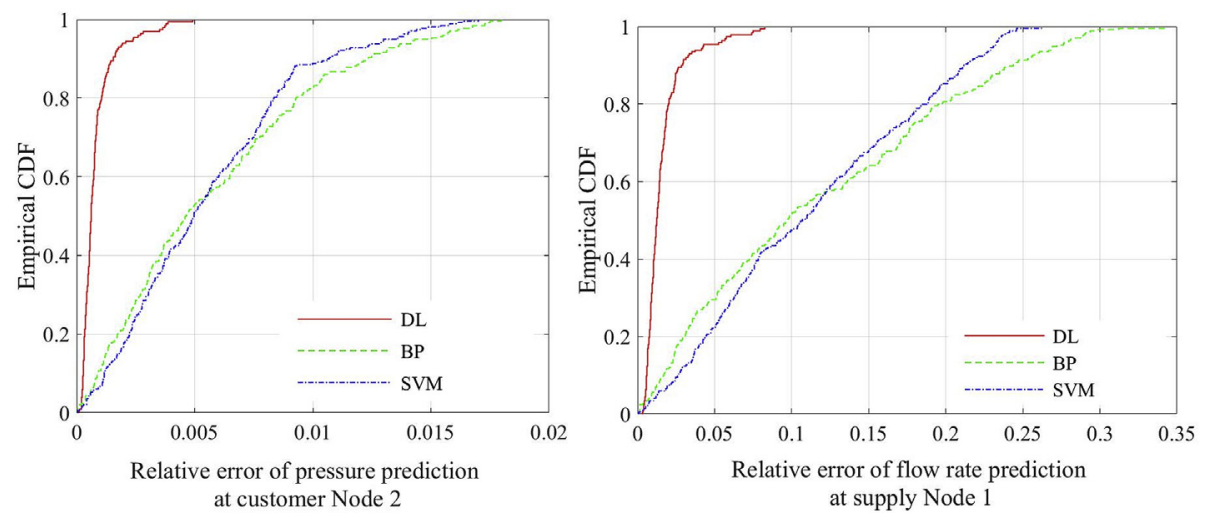

Fig. 10. Performance comparison based on the empirical CDF of the relative error of prediction results ( $5 \mathrm{~h}$ prediction).
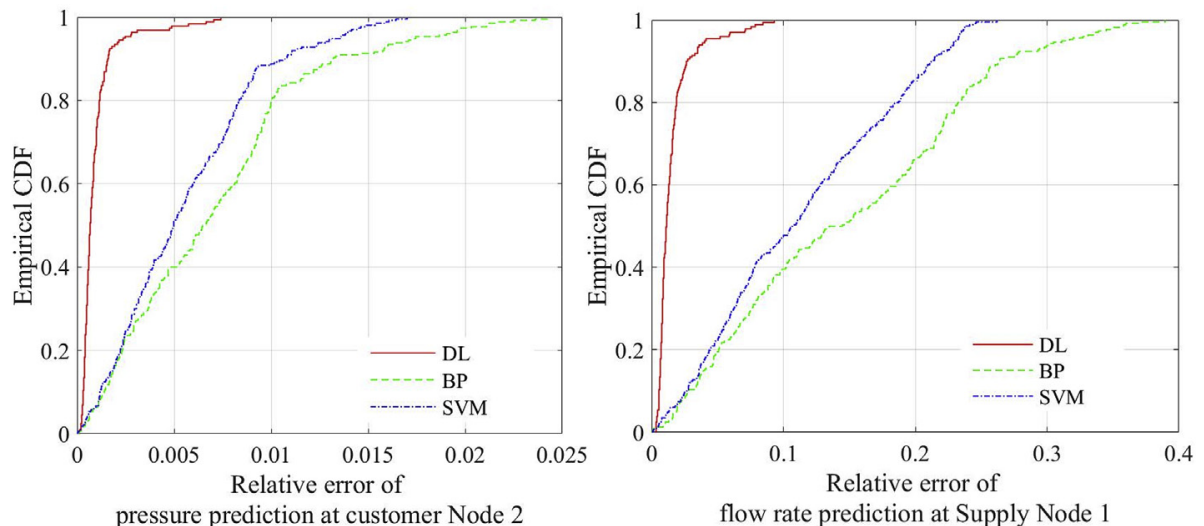

Fig. 11. Performance comparison based on the empirical CDF of the relative error of prediction results ( $9 \mathrm{~h}$ prediction).

input data). In this case, the artificial noises are added on the numerical simulation results, to analyze the robustness of the deep learning model under the noises from the data collection process. The corresponding empirical CDF results of the predictions are shown in Fig. 15. In the
Figure, we can observe that the predictions are accurate with the different levels of noise considered. However, there is a relatively significant jump of the results from $1.0 \%$ noise to $1.5 \%$ noise, so that to reduce noise below the level of $1.0 \%$ could be effective for prediction

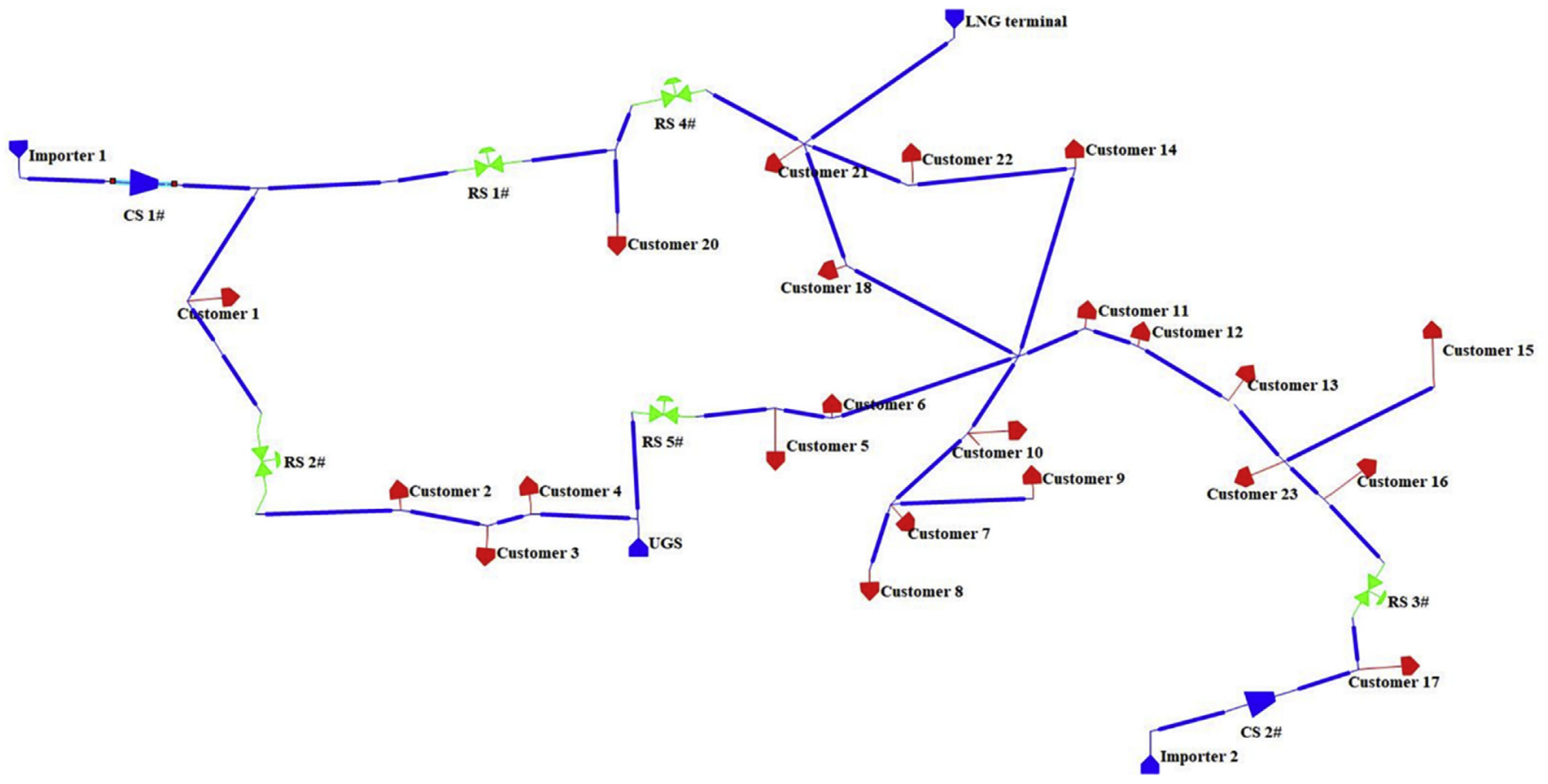

Fig. 12. Layout of the complex natural gas pipeline network system. 
Table 3

Prediction performances comparison for the three different sets of input data and with reference to pipeline average gas flow rates predictions $\left(\mathrm{Nm}^{3} / \mathrm{s}\right)$.

\begin{tabular}{|c|c|c|c|c|c|c|}
\hline Task & \multicolumn{2}{|c|}{ Not-preprocessed data } & \multicolumn{2}{|c|}{ Preprocessed data } & \multicolumn{2}{|c|}{ No pressure input } \\
\hline $5 \mathrm{~h}$ prediction & 0.3107 & 0.4520 & 0.3076 & 0.4499 & 1.1463 & 1.9510 \\
\hline
\end{tabular}

Table 4

Prediction performances comparison for the three different sets of input data and with reference to pressures predictions (Pa).

\begin{tabular}{|c|c|c|c|c|c|c|}
\hline Task & \multicolumn{2}{|c|}{ Not-preprocessed data } & \multicolumn{2}{|c|}{ Preprocessed data } & \multicolumn{2}{|c|}{ No pressure input } \\
\hline $5 \mathrm{~h}$ prediction & 1290.74 & 1606.79 & 1254.31 & 1579.92 & 7349.60 & 8597.88 \\
\hline
\end{tabular}
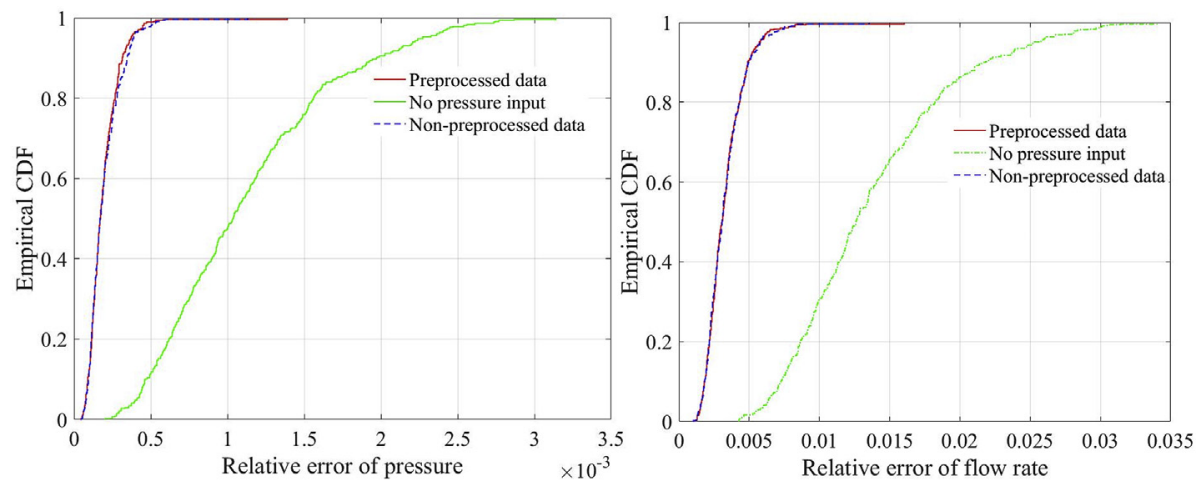

Fig. 13. Performances comparison based on the empirical CDF of the relative error of the prediction results, based on different sets of input data.

Table 5

Prediction performances comparison based on different time lengths of prediction.

\begin{tabular}{lllll}
\hline Task & \multicolumn{2}{l}{ Pressure (Pa) } & \multicolumn{2}{l}{ Flow rate $\left(\mathrm{Nm}^{3} / \mathrm{s}\right)$} \\
\cline { 2 - 5 } & MAE & RMSE & MAE & RMSE \\
\hline 2 h prediction & 1145.42 & 1462.24 & 0.2340 & 0.3622 \\
5 h prediction & 1254.36 & 1579.88 & 0.3076 & 0.4499 \\
8 h prediction & 1571.41 & 1984.27 & 0.3527 & 0.5188 \\
10 h prediction & 1662.94 & 2108.31 & 0.3656 & 0.5373 \\
15 h prediction & 2843.90 & 3664.89 & 0.5828 & 0.8656 \\
\hline
\end{tabular}

accuracy in this case.

The deep learning model is also capable to effectively predict the system conditions in abnormal scenarios. In general, crises of natural gas supply security result from abnormal increases of demands and/or

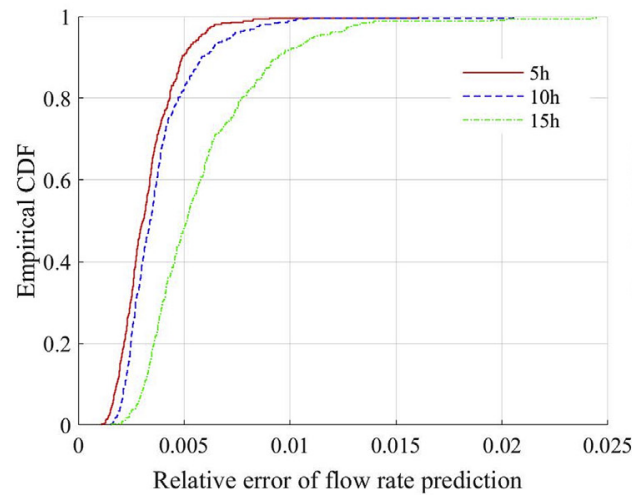

Table 6

Prediction performances analysis for Scenarios 1-3.

\begin{tabular}{lllllll}
\hline Task & \multicolumn{2}{l}{ Scenario 1} & \multicolumn{3}{l}{ Scenario 2 } & \multicolumn{2}{l}{ Scenario 3 } \\
\cline { 2 - 6 } & MAE & RMSE & MAE & RMSE & MAE & RMSE \\
\hline $\begin{array}{c}\text { Pressure } \\
\text { prediction } \\
\quad \text { (Pa) }\end{array}$ & 3380.55 & 4509.76 & 2792.36 & 3571.59 & 3633.47 & 4519.46 \\
$\begin{array}{c}\text { Flow rate } \\
\text { prediction } \\
\left(\mathrm{Nm}^{3} / \mathrm{s}\right)\end{array}$ & 1.09 & 1.67 & 0.72 & 1.00 & 1.03 & 1.49 \\
$\begin{array}{c}\text { Supply prediction } \\
\left(\mathrm{Nm}^{3} / \mathrm{s}\right)\end{array}$ & 2.41 & 3.02 & 1.93 & 2.43 & 2.14 & 2.47 \\
\hline
\end{tabular}

decreases in the capacity of sources. To analyze this, predictions for $5 \mathrm{~h}$ are performed under three abnormal scenarios:

Scenario 1: Abnormal demands increases at Customers 4 and 23.

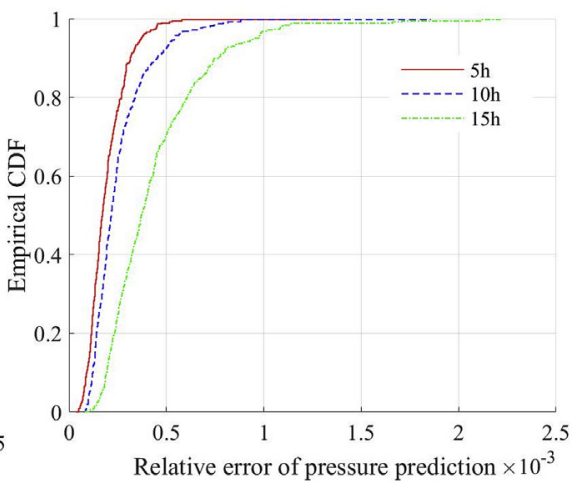

Fig. 14. Performances comparison based on the empirical CDFs of the relative error values of the predictions, based on different time lengths of predictions. 

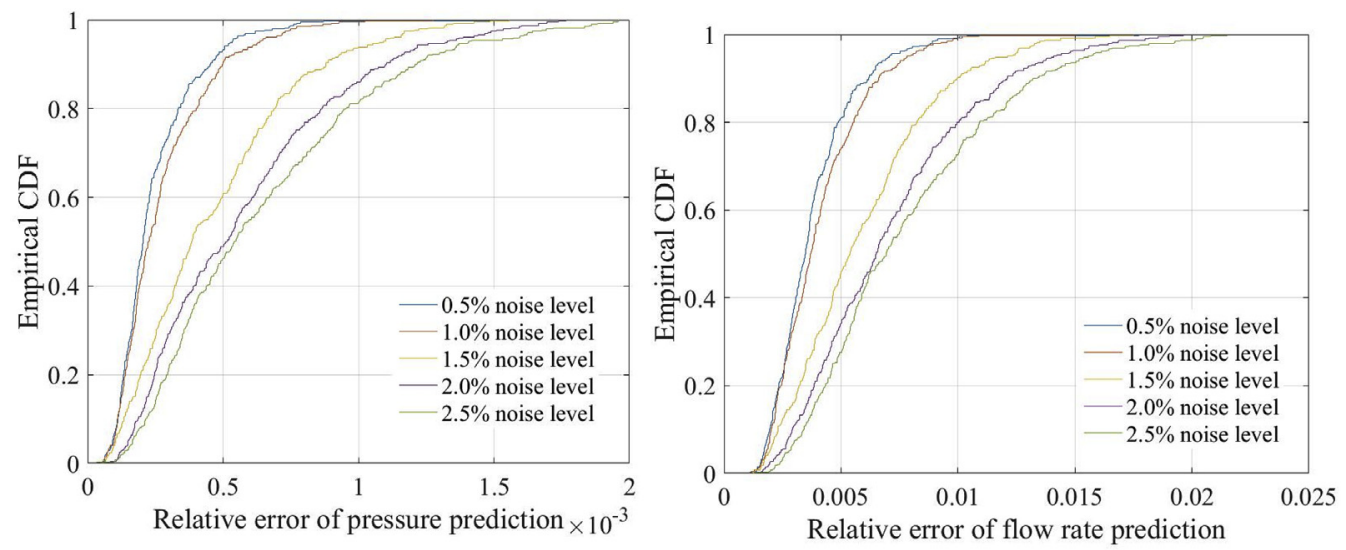

Fig. 15. Performances comparison based on the empirical CDFs of the relative error values of the predictions, based on under different levels of noises.
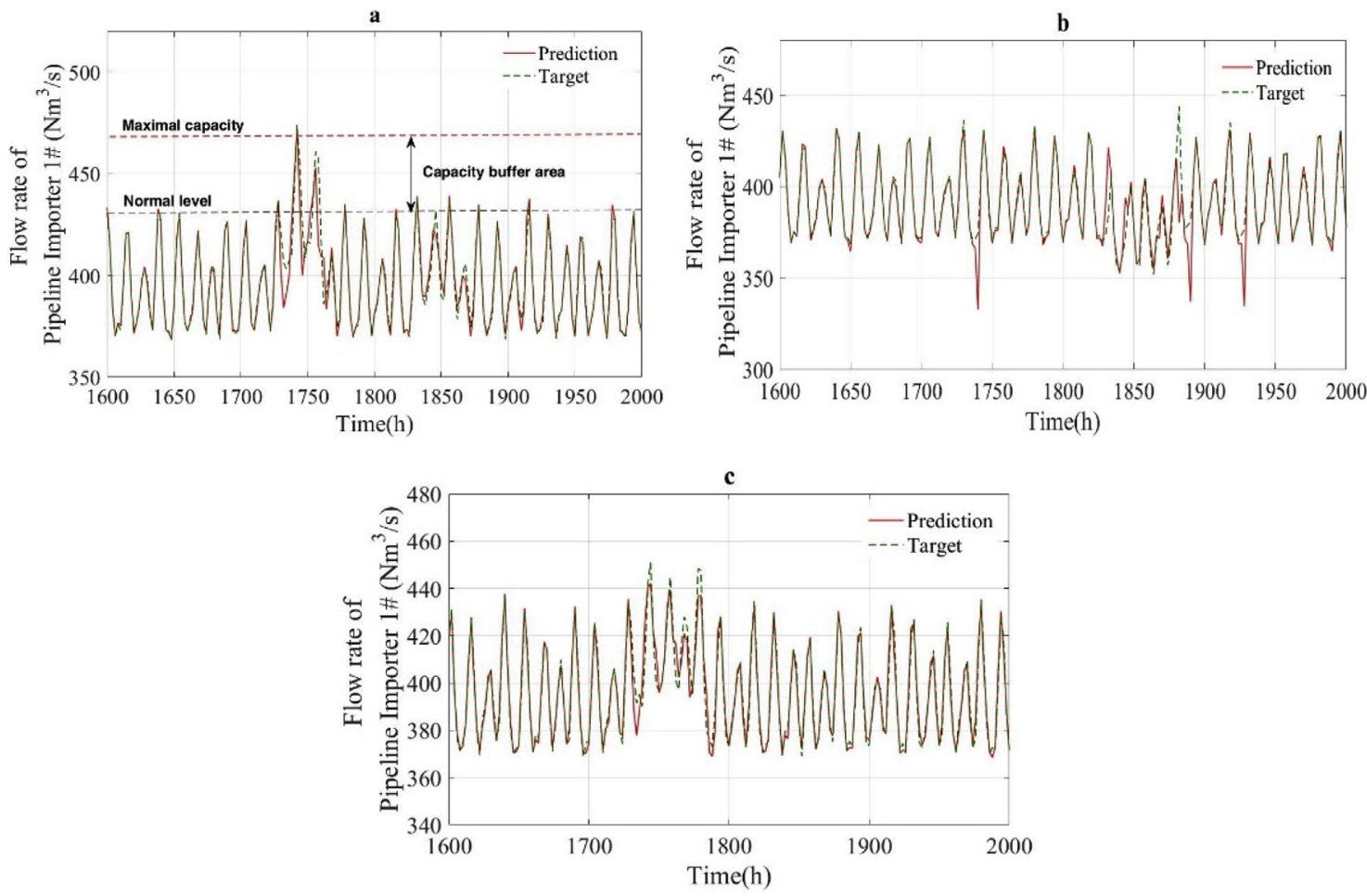

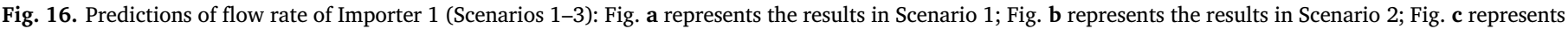
the results in Scenario 3.

The demand of Customer 4 suddenly increases to $250 \%$ (from 1720 to $1740 \mathrm{~h}$ ) and the demand of Customer 23 suddenly increases to $300 \%$ (from 1820 to $1850 \mathrm{~h}$ ).

Scenario 2: Sudden pressure decrease at Pipeline Importer 1 . The pressure of Importer 1 suddenly degenerates to $6.9 \mathrm{MPa}$, from the normal value of $7 \mathrm{MPa}$ (from 1820 to $1870 \mathrm{~h}$ ).

Scenario 3: Sudden supply capacity degeneration of the UGS. The supply capacity of the UGS suddenly decreases to $20 \%$ (from 1720 to $1770 \mathrm{~h})$.

Considering the learning principles of the deep neural network, we introduce two changes when generating the training data sets for each abnormal scenario. For example, in Scenario 2, two pressure drops of Pipeline Importer 1 , namely a $50 \mathrm{~h}$ ' pressure drop to $6.89 \mathrm{MPa}$ and a $50 \mathrm{~h}$ ' pressure drop to $6.92 \mathrm{MPa}$, are introduced in the system simulation.

The results of predictions of the system conditions are illustrated from the different perspectives, i.e. gas flow rates of the main suppliers (pressure controlled), pressures at demand sites, pressures of the suppliers (flow rate controlled), flow rates of important pipelines, which managers and operators are more concerned with in practice (Pambour et al., 2016b). For every perspective, typical results are analyzed in detail below.

The results of Scenarios 1-3 are presented in Figs. 16-20.

We observe that the evolution of the pressures and loads for the suppliers, delivery point and the important connection pipeline are accurately predicted, which means that the deep learning model is able to provide timely information to support operation and management in abnormal conditions.

Figs. 15 and 16 show that the deep learning is able to predict the responses of the two suppliers in abnormal scenarios. For intuitive application in practice, the concept of capacity buffer area is introduced in the Figs. By comparing the predictions and the maximal capacities, supply security can be predicted, which can be helpful to operators to respond in time and make efficient use of supply capacities. 

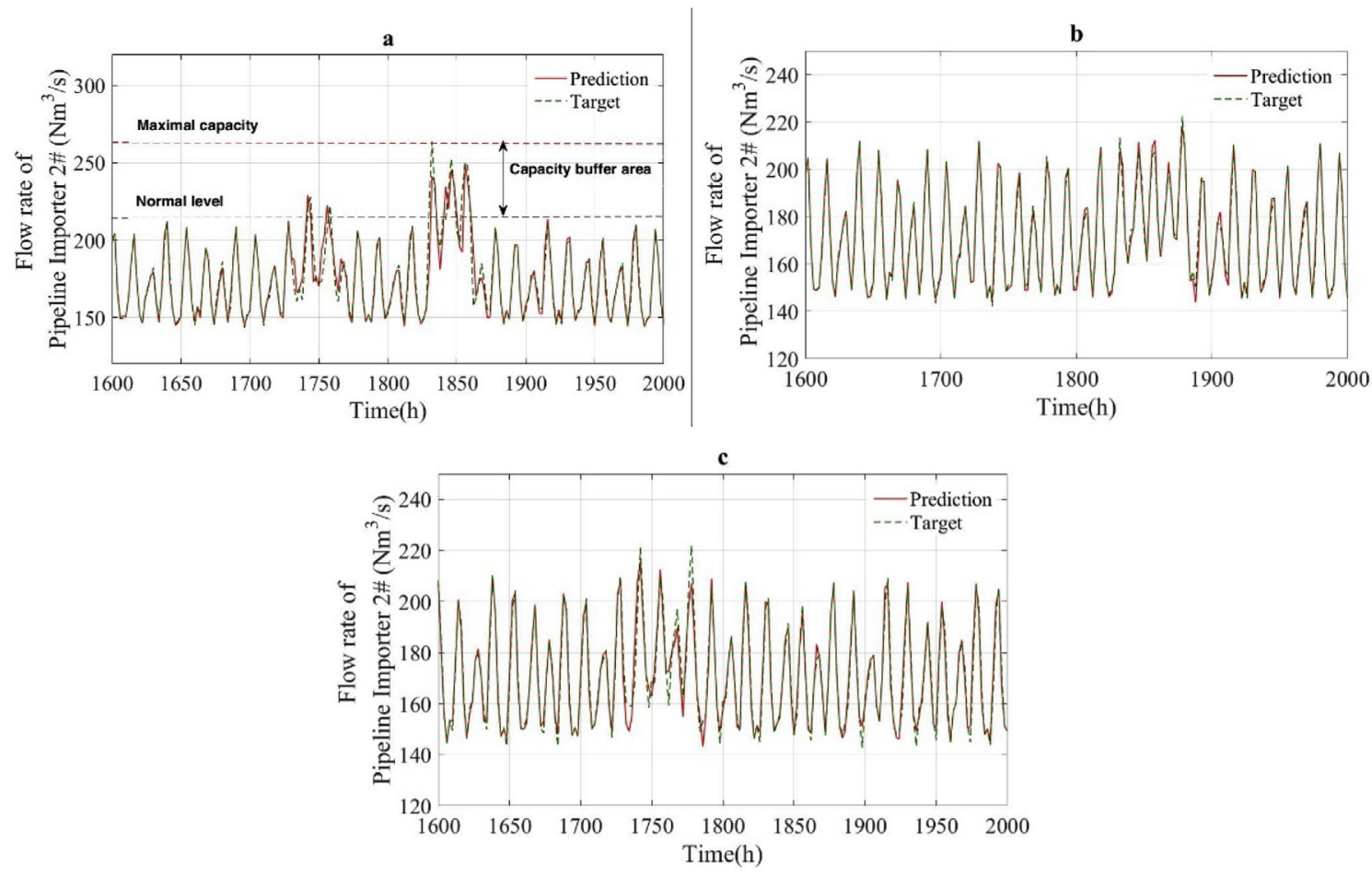

Fig. 17. Predictions of flow rate of Importer 2 (Scenarios 1-3): Fig. a represents the results in Scenario 1; Fig. b represents the results in Scenario 2; Fig. c represents the results in Scenario 3.
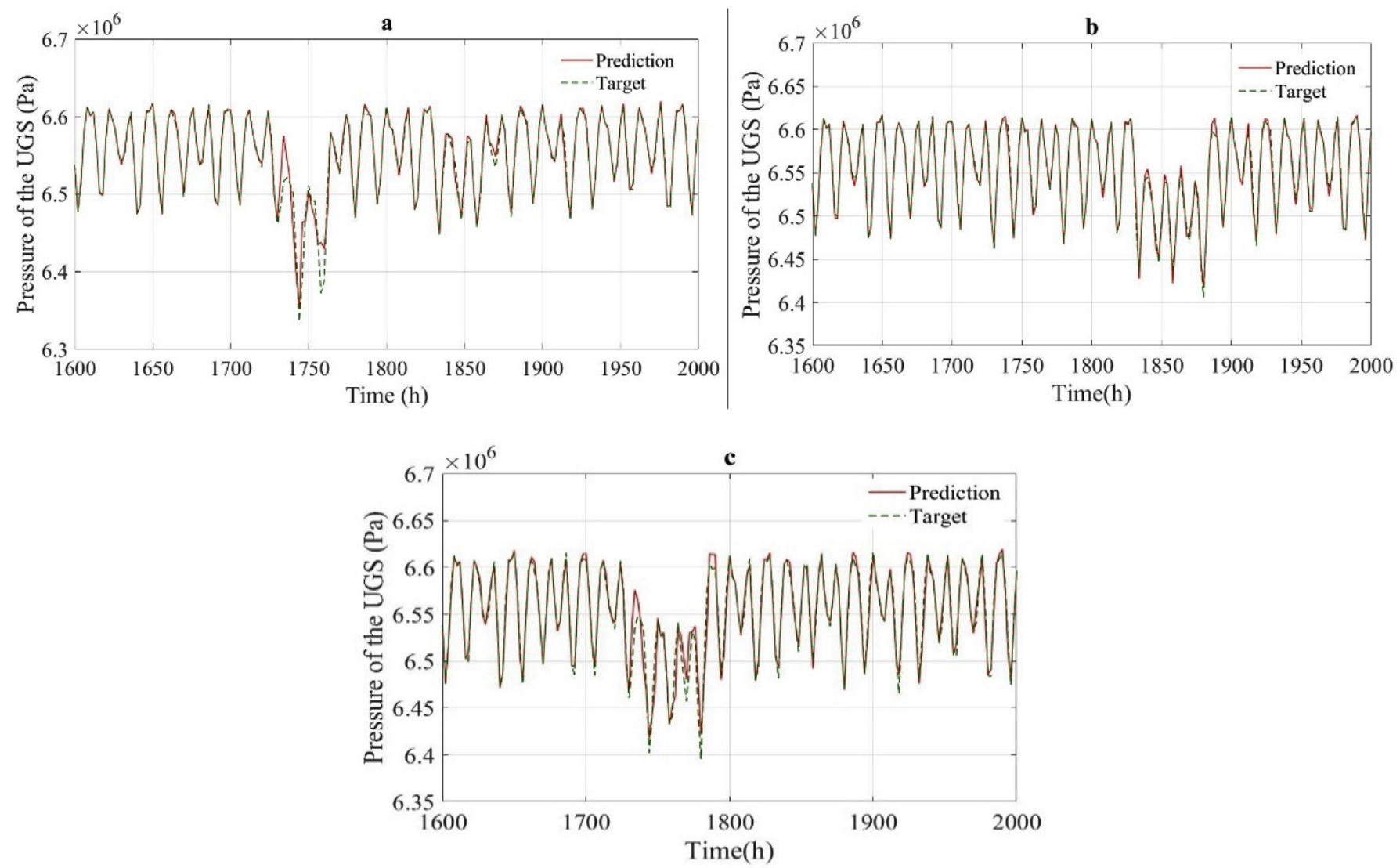

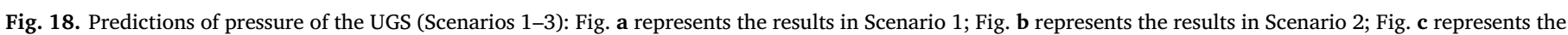
results in Scenario 3. 

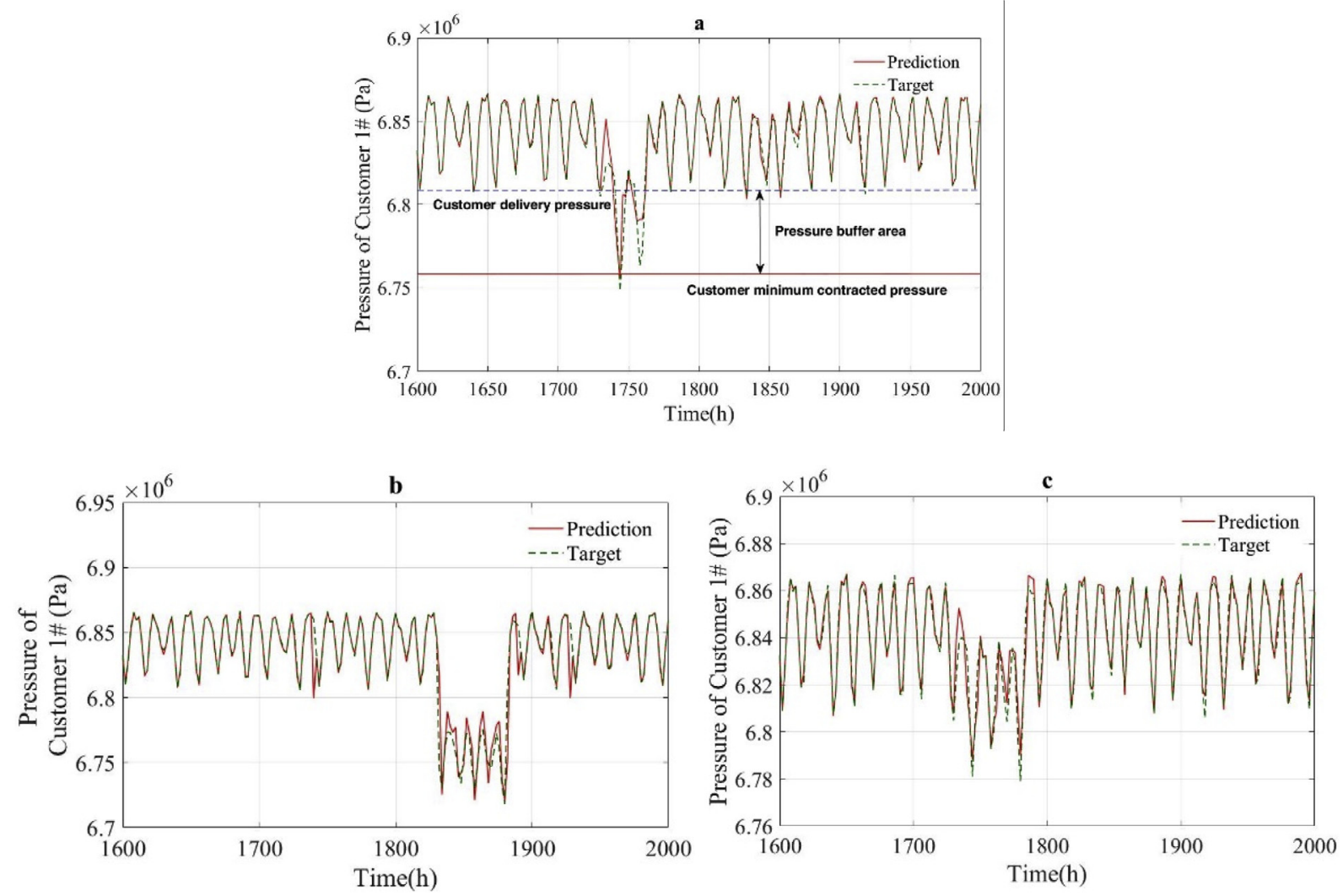

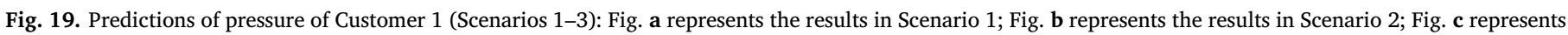
the results in Scenario 3.
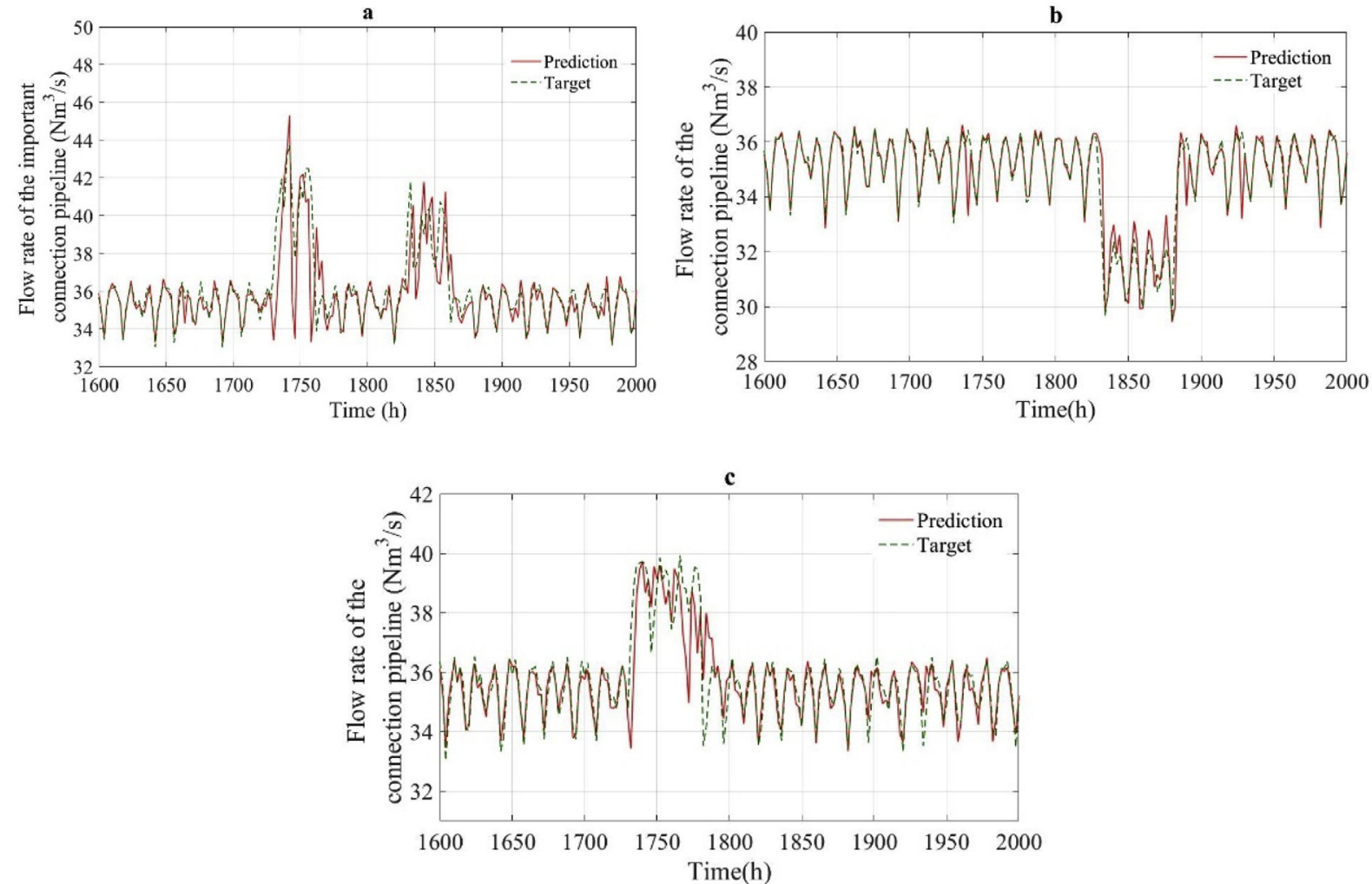

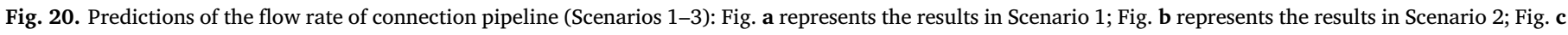
represents the results in Scenario 3. 
Fig. 18 presents the predictions of the pressure values of the UGS. In the Figs, we can observe that the pressure drops, which help maintaining stable supply of gas under abnormal conditions, are effectively captured by the deep learning model. This type of ability is important for estimating the conditions of critical components and making timely preventing decisions, to ensure the reliability of supply of natural gas.

Pressure buffer, which is defined as the difference between the pressure of the real-time delivery pressure and the contractual delivery, is one of the most concerned issues of natural gas supply security. Fig. 19 shows that the transient behaviors of the pressure buffer under different abnormal scenarios are captured by the deep learning model. Hence, this can provide a powerful method to predict security of supply of the customers and to guarantee a high level of customer satisfaction with a low consumption of energy.

The profiles in Fig. 20 represent the flow rate through the connection pipeline between Customer 11 and the multi-junction node, which is important for the supply security considering congestion problems. By comparing the true and predicted flow rates, we find that the predictions can effectively reflect the true future trends of gas flows, which indicates that the deep learning model can be used to optimize the plan of gas transmission according to changing conditions and ensure a more reliable operation and supply.

However, by comparing the prediction performances in Table 6 and Tables 3-5, we can observe a degeneration of accuracy after abnormal changes occur. This is because only two similar changes are introduced in the training data and the features of the complicated dynamic system properties under these abnormal conditions have not been learned well enough. In practice, more data can be used as training data and the data sets can be continuously updated from the fault.

\section{Conclusion}

In this paper, we have presented a framework based on deep learning, for the prediction of the operation conditions of natural gas pipeline networks. A prediction model, based on real-time data, is developed by combining a SAE model with a regression layer. To reduce the problem size, structural controllability theory has been applied for selecting the input data most relevant for prediction, and a data window has been used to create a proper "memory" for the deep learning model.

The accuracy of prediction of the deep learning model has been verified by benchmarking against BP neural network and SVM, on a case of a triangle gas pipeline network. The effectiveness of the proposed framework has been analyzed and verified from multiple perspectives, i.e. type of input, length of prediction time and level of noise, with respect to a relatively complex gas pipeline network. To analyze the deep learning method for abnormal conditions, three scenarios have been considered. The results show that the proposed deep learning model is able to accurately capture the evolution of system conditions under different abnormal changes. The average accuracy of prediction of the working condition within $15 \mathrm{~h}$ is higher than 0.99 . Besides, the deep learning model presents robust performances and is able to maintain the high level of accuracy even under a relatively high level of noises (from $\pm 0.5 \%$ to $\pm 2.5 \%$ of the nominal values of input data). Also, the case study includes the compressor stations, the LNG terminals and the UGS, whose working conditions are changing according to predefined rules, and the results show that the developed method can make good predictions with changing working pressures of the LNG terminals and the UGS.

In future work, we will further improve the deep learning model, considering other relevant factors in input and perspectives, e.g., uncertainty management, demand response, dynamic programming, etc. Also, some other powerful intelligence methods, such as ANFIS, advanced genetic programming, RNN-structured neural networks and so on, will be considered in our future work, and their performance will be compared with the proposed deep learning method. The relatively large errors in pressure prediction, caused by the de-normalizing process, calls for a more effective data-processing method.

\section{Acknowledgement}

This work is supported by National Natural Science Foundation of China [grant number 51134006]. The authors wish to acknowledge the kind help of Wei Wang and Mingjing $\mathrm{Xu}$, the $\mathrm{Ph}$. D students at Dipartimento di Energia, Politecnico di Milano.

\section{Nomenclature}

$a, b, c \quad$ constants of Mackey-Glass model SAE stacked auto-encoder BP backward propagation SVM support vector machine B vector of encoding bias UGS underground gas storage

C vector of decoding bias $\mathbf{W}_{1}$ weight matrix

$E \quad$ error of reconstruction $\mathbf{W}_{2}$ decoding matrix

$H \quad$ number of hidden units $\boldsymbol{x}$ input vector of an auto-encoder

$K L \quad$ Kullback-Leibler divergence $y$ vector of the hidden representation

LNG liquified natural gas $\mathbf{z}$ output vector of an auto-encoder

MAE mean absolute error $\theta$ model parameter

MRE mean relative error $\varphi$ weight of the sparsity term

RMSE root-mean-square error $\tau_{M}$ time delay parameter

$S \quad$ designed length of the input part

\section{References}

Azadeh, A., et al., 2016. A unique adaptive neuro fuzzy inference system for optimum decision making process in a natural gas transmission unit. J. Nat. Gas Sci. Eng. 34, 472-485. Available at: https://www.sciencedirect.com/science/article/pii/ S187551001630436X [Accessed March 6, 2018].

Behbahani-Nejad, M., Bagheri, A., 2010. The accuracy and efficiency of a MATLABSimulink library for transient flow simulation of gas pipelines and networks. J. Petrol. Sci. Eng. 70 (3-4), 256-265.

Bengio, Y., et al., 2007. Greedy layer-wise training of deep networks. Adv. Neural Inf. Process. Syst. 19 (1), 153. Available at: http://papers.nips.cc/paper/3048-greedylayer-wise-training-of-deep-networks.pdf [Accessed January 8, 2018].

Chertkov, M., Backhaus, S., Lebedev, V., 2015. Cascading of fluctuations in interdependent energy infrastructures: gas-grid coupling. Appl. Energy 160, 541-551.

Chiang, N.Y., Zavala, V.M., 2016. Large-scale optimal control of interconnected natural gas and electrical transmission systems. Appl. Energy 168, 226-235.

Durgut, İ., Leblebicioğlu, M.K., 2016. State estimation of transient flow in gas pipelines by a Kalman filter-based estimator. J. Nat. Gas Sci. Eng. 35, 189-196.

Evermann, J., Rehse, J.-R., Fettke, P., 2017. Predicting process behaviour using deep learning. Decis. Support Syst. 100, 129-140. Available at: http://linkinghub.elsevier.

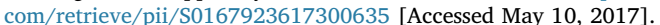

Faertes, D., et al., 2010. Reliability modelling: petrobras 2010 integrated gas supply chain. In: In 2010 8th International Pipeline Conference, vol. 4. ASME, pp. 497-505. Available at: http://proceedings.asmedigitalcollection.asme.org/proceeding.aspx? articleid $=1615244$ [Accessed November 25, 2016].

Fang, Y.-P., Zio, E., 2013. Unsupervised spectral clustering for hierarchical modelling and criticality analysis of complex networks. Reliab. Eng. Syst. Saf. 116, 64-74. Available at: https://ac.els-cdn.com/S0951832013000562/1-s2.0-S0951832013000562-main. pdf?_tid = 4981326a-f479-11e7-8c53-00000aab0f27\&acdnat $=1515418922$ e72fb1de27747b07430531e56d9404c8 [Accessed January 8, 2018].

Fasihizadeh, M., Sefti, M.V., Torbati, H.M., 2014. Improving gas transmission networks operation using simulation algorithms: case study of the National Iranian Gas Network. J. Nat. Gas Sci. Eng. 20, 319-327.

Güler, I., Übeyli, E.D., 2006. A recurrent neural network classifier for Doppler ultrasound blood flow signals. Pattern Recogn. Lett. 27 (13), 1560-1571. Available at: https:// ac.els-cdn.com/S0167865506000730/1-s2.0-S0167865506000730-main.pdf?_tid= 3f81f4c9-2e13-4d2d-b061-8cce9d771567\&acdnat = 1527495735 1335b9b71c37446de7042f63bfa7677d [Accessed May 28, 2018].

Güler, N.F., Übeyli, E.D., Güler, I., 2005. Recurrent neural networks employing Lyapunov exponents for EEG signals classification. Expert Syst. Appl. 29 (3), 506-514. Available at: https://ac.els-cdn.com/S0957417405000679/1-s2.0S0957417405000679-main.pdf? tid =078d2a06-1a4f-4714-87ef-da84031974f8\& acdnat $=1527495686 \_3 a 8 f 01196$ bf9a685be8bef11766d259c [Accessed May 28, 2018].

Han, F., Prodan, I., Zio, E., 2015. A framework of model predictive control for the safety analysis of an electric power microgrid. In: Safety and Reliability of Complex Engineered Systems. CRC Press, pp. 1553-1564. Available at: http://www. crcnetbase.com/doi/10.1201/b19094-203 [Accessed September 21, 2017].

Hegde, C., Gray, K.E., 2017. Use of machine learning and data analytics to increase drilling efficiency for nearby wells. J. Nat. Gas Sci. Eng. 40, 327-335. Available at: https://www.sciencedirect.com/science/article/pii/S1875510017300641 [Accessed 
March 6, 2018].

Hinton, G.E., Osindero, S., Teh, Y.-W., 2006. A fast learning algorithm for deep belief nets. Neural Comput. 18 (7), 1527-1554. Available at: http://www.mitpressjournals. org/doi/10.1162/neco.2006.18.7.1527 [Accessed January 8, 2018].

Hinton, G.E., Salakhutdinov, R.R., 2006. Reducing the dimensionality of data with neural networks. Science 313 (5786), 504-507. Available at: http://www.ncbi.nlm.nih. gov/pubmed/16873662 [Accessed November 8, 2017].

$\mathrm{Hu}$, J., et al., 2010. An integrated method for safety pre-warning of complex system. Saf. Sci. 48 (5), 580-597. Available at: http://ac.els-cdn.com/S0925753510000135/1s2.0-S0925753510000135-main.pdf?_tid = fdb68690-992f-11e7-912f-00000aab0f6b \&acdnat $=1505381885$ _0f990dff1af8cd79ca199159a82f5816 [Accessed September 14, 2017].

Hu, Y., et al., 2017. A systematic semi-supervised self-adaptable fault diagnostics approach in an evolving environment. Mech. Syst. Signal Process. 88, 413-427. Available at: https://ac.els-cdn.com/S0888327016304666/1-s2.0S0888327016304666-main.pdf? tid = 0f91a848-f481-11e7-91f2-00000aab0f02\& acdnat $=1515422261$ 7e1f99d74fc21b5b16ceca5890b86994 [Accessed January 8, 2018].

Kalantari-Dahaghi, A., Mohaghegh, S., Esmaili, S., 2015. Coupling numerical simulation and machine learning to model shale gas production at different time resolutions. J. Nat. Gas Sci. Eng. 25, 380-392. Available at: https://www.sciencedirect.com/ science/article/pii/S1875510015001651 [Accessed March 6, 2018].

Kalman, R.E., 1963. Mathematical description of linear dynamical systems. Journal of the Society for Industrial and Applied Mathematics Series A Control 1 (2), 152-192. Available at: http://epubs.siam.org/doi/10.1137/0301010 [Accessed January 8, 2018].

Karadede, Y., Ozdemir, G., Aydemir, E., 2017. Breeder hybrid algorithm approach for natural gas demand forecasting model. Energy 141, 1269-1284. Available at: https://ac.els-cdn.com/S0360544217316511/1-s2.0-S0360544217316511-main. pdf $?$ tid $=$ f5b0ad0c-f476-11e7-8d68-00000aab0f01\&acdnat $=1515417922$ e5a4c1a66c309c4c37f3acfcdb540b8c [Accessed January 8, 2018].

Kong, X., et al., 2018. Deep learning hybrid method for islanding detection in distributed generation. Appl. Energy 210, 776-785.

Lashkarbolooki, M., et al., 2013. Fluid Phase Equilibria Investigating vapor-liquid equilibria of binary mixtures containing supercritical or near-critical carbon dioxide and a cyclic compound using cascade neural network. Fluid Phase Equil. 343, 24-29. Available at: https://ac.els-cdn.com/S0378381213000381/1-s2.0S0378381213000381-main.pdf? tid = 3529af45-784e-432f-9b4a-8f4afe643a3e \& acdnat $=1527495749 \_$adc4fcfa67f9b2cbd2debdbcae7c9b8f [Accessed May 28, 2018].

Leitold, D., Vathy-Fogarassy, Á., Abonyi, J., 2017. Controllability and observability in complex networks - the effect of connection types. Sci. Rep. 7 (1), 151. Available at: http://www.nature.com/articles/s41598-017-00160-5 [Accessed March 23, 2017].

Liu, Y.-Y., Slotine, J.-J., Barabási, A.-L., 2011. Controllability of complex networks. Nature 473 (7346), 167-173. Available at: http://www.nature.com/doifinder/10. 1038/nature10011.

Lou, S., et al., 2016. Prediction of diffuse solar irradiance using machine learning and multivariable regression. Appl. Energy 181, 367-374. Available at: https://ac.els cdn.com/S0306261916311916/1-s2.0-S0306261916311916-main.pdf?_tid = e7ec3226-f477-11e7-9a3e-00000aab0f6c\&acdnat $=1515418329$ 4123bcd43f927955a3eca0a32d922b26 [Accessed January 8, 2018].

Lv, Y., et al., 2015. Traffic flow prediction with big data: a deep learning approach. IEEE Trans. Intell. Transport. Syst. 16 (2), 865-873. Available at: http://ieeexplore.ieee. org/lpdocs/epic03/wrapper.htm?arnumber $=6894591$ [Accessed November 8 , 2017].

Madoliat, R., Khanmirza, E., Moetamedzadeh, H.R., 2016. Transient simulation of gas pipeline networks using intelligent methods. J. Nat. Gas Sci. Eng. 29, 517-529.

Pambour, K.A., Bolado-Lavin, R., Dijkema, G.P.J., 2016a. An integrated transient model for simulating the operation of natural gas transport systems. J. Nat. Gas Sci. Eng. 28, 672-690.

Pambour, K.A., Bolado-Lavin, R., Dijkema, G.P.J., 2016b. An integrated transient model for simulating the operation of natural gas transport systems. J. Nat. Gas Sci. Eng. 28, 672-690. Available at: http://www.sciencedirect.com/science/article/pii/ S1875510015302766.

Panapakidis, I.P., Dagoumas, A.S., 2017. Day-ahead natural gas demand forecasting based on the combination of wavelet transform and ANFIS/genetic algorithm/neural network model. Energy 118, 231-245. Available at: https://ac.els-cdn.com/ S0360544216318382/1-s2.0-S0360544216318382-main.pdf?_tid = de83b4b2-f47611e7-a2e6-00000aab0f6c\&acdnat $=1515417883$ 1356f4854577ee9c3d511f73490a09eb [Accessed January 8, 2018].

Pooyan, N., et al., 2015. Simultaneous Fault Diagnosis using multi class support vector machine in a Dew Point process. J. Nat. Gas Sci. Eng. 23, 373-379. Available at: https://www.sciencedirect.com/science/article/pii/S1875510015000566 [Accessed March 6, 2018]

Qiu, J., et al., 2015. The early-warning model of equipment chain in gas pipeline based on DNN-HMM. J. Nat. Gas Sci. Eng. 27, 1710-1722. Available at: http://ac.els-cdn. com/S1875510015302407/1-s2.0-S1875510015302407-main.pdf?_tid = 3ded82fa- 992f-11e7-82d3-00000aab0f6c\&acdnat $=1505381564$

b4f384c73defb978c5bfb48574dc6b93 [Accessed September 14, 2017].

Sharma, V., et al., 2016. Short term solar irradiance forecasting using a mixed wavelet neural network. Renew. Energy 90, 481-492. Available at: https://ac.els-cdn.com/ S0960148116300209/1-s2.0-S0960148116300209-main.pdf?_tid=79f71d90-f46211e7-bbb0-00000aab0f02\&acdnat $=1515409125$ f3bee543c5273e09a03aac65577b3ab8 [Accessed January 8, 2018].

Shields, R.W., Pearson, J.B., 1976. Structural controllability of multiinput linear systems. IEEE Trans. Automat. Contr. 21 (2), 203-212.

Tan, P., et al., 2016. Modeling and reduction of NOX emissions for a 700 MW coal-fired boiler with the advanced machine learning method. Energy 94, 672-679. Available at: https://ac.els-cdn.com/S0360544215015558/1-s2.0-S0360544215015558-main. pdf? tid = 7c0abfc2-f478-11e7-b288-00000aab0f01\&acdnat $=1515418577$ c0bd83c682dd54985c28090f7dd56718 [Accessed January 8, 2018].

Turati, P., Pedroni, N., Zio, E., 2017. Simulation-based exploration of high-dimensional system models for identifying unexpected events. Reliab. Eng. Syst. Saf. 165, 317-330. Available at: https://ac.els-cdn.com/S0951832016302964/1-s2.0S0951832016302964-main.pdf?_tid=01096260-f482-11e7-a7f6-00000aab0f02\& acdnat $=1515422666 \_$ea03b7fe5afa6b686e0a5a779645a4dc [Accessed January 8, 2018].

Uilhoorn, F.E., 2017. Comparison of Bayesian estimation methods for modeling flow transients in gas pipelines. J. Nat. Gas Sci. Eng. 38, 159-170. Available at: http://ac els-cdn.com/S1875510016309076/1-s2.0-S1875510016309076-main.pdf?_tid = 41f201f6-0806-11e7-8e22-00000aacb35e\&acdnat $=1489421042$ b82e4a6250b5c3573b18e275cba1f47f [Accessed March 13, 2017].

Vaferi, B., Eslamloueyan, R., Ayatollahi, S., 2015. Application of recurrent networks to classification of oil reservoir models in well-testing analysis. Energy Sources, Part A Recovery, Util. Environ. Eff. 37 (2), 174-180. Available at: http://www.tandfonline. $\mathrm{com} /$ action/journalinformation? journalCode = ueso20 [Accessed May 28, 2018].

Wang, P., et al., 2015. Comparison study on the accuracy and efficiency of the four forms of hydraulic equation of a natural gas pipeline based on linearized solution. J. Nat. Gas Sci. Eng. 22, 235-244. Available at: https://www.sciencedirect.com/science/ article/pii/S1875510014003746 [Accessed March 6, 2018].

Wang, P., et al., 2018. Fast method for the hydraulic simulation of natural gas pipeline networks based on the divide-and-conquer approach. J. Nat. Gas Sci. Eng. 50, 55-63. Available at: https://www.sciencedirect.com/science/article/pii/ S1875510017304390 [Accessed March 6, 2018].

Wang, Z., et al., 2016. A power flow based model for the analysis of vulnerability in power networks. Phys. Stat. Mech. Appl. 460, 105-115. Available at: http://www. sciencedirect.com/science/article/pii/S0378437116301868 [Accessed April 18, 2017].

Xiong, R., Cao, J., Yu, Q., 2018. Reinforcement learning-based real-time power management for hybrid energy storage system in the plug-in hybrid electric vehicle. Appl. Energy 211, 538-548. Available at: https://ac.els-cdn.com/S0306261917316707/1 s2.0-S0306261917316707-main.pdf? tid = 5ae9accc-f478-11e7-9f2c-00000aab0f26\& acdnat $=1515418522 \_11$ ba58033c0231e128e7a668551e9027 [Accessed January 8, 2018].

Xue, P., et al., 2017. Fault detection and operation optimization in district heating substations based on data mining techniques. Appl. Energy 205, 926-940. Available at: https://ac.els-cdn.com/S0306261917310401/1-s2.0-S0306261917310401-main. pdf? tid $=$ dc5404ae-f45c-11e7-b100-00000aab0f26\&acdnat $=1515406713$ 8b6902c6a2e26c15fbc4c803d58bbab1 [Accessed January 8, 2018].

Yu, F., Xu, X., 2014. A short-term load forecasting model of natural gas based on optimized genetic algorithm and improved BP neural network. Appl. Energy 134, 102-113. Available at: https://ac els-cdn.com/S0306261914007958/1-s2.0S0306261914007958-main.pdf?_tid = 961cc93a-f47a-11e7-99c3-00000aab0f6c\& acdnat $=1515419480 \_d 70 f 0 f 2 b f 7 e 18$ e9acd282815e2e41d28 [Accessed January 8, 2018].

Zhang, D., et al., 2016a. ScienceDirect some improvements of particle filtering based prognosis for PEM fuel cells. IFAC-PapersOnLine 49, 162-167. Available at: www. sciencedirect.com [Accessed October 31, 2017].

Zhang, X., Wu, C., Zuo, L., 2016b. Minimizing fuel consumption of a gas pipeline in transient states by dynamic programming. J. Nat. Gas Sci. Eng. 28, 193-203. Available at: https://www.sciencedirect.com/science/article/pii/ S1875510015302754 [Accessed March 6, 2018].

Zhou, D., et al., 2017. Degradation prediction of PEM fuel cell using a moving window based hybrid prognostic approach. Energy 138, 1175-1186. Available at: https://ac. els-cdn.com/S0360544217312732/1-s2.0-S0360544217312732-main.pdf?.tid = ab379506-cabe-11e7-9109-00000aacb35e\&acdnat $=1510830772$ 7b10981fe11567098df51d0d81f03644 [Accessed November 16, 2017].

Zio, E., 2016. Challenges in the vulnerability and risk analysis of critical infrastructures. Reliab. Eng. Syst. Saf. 152, 137-150. Available at: https://doi.org/10.1016/j.ress. 2016.02.009.

Zio, E., Di Maio, F., 2010. A data-driven fuzzy approach for predicting the remaining useful life in dynamic failure scenarios of a nuclear system. Reliab. Eng. Syst. Saf. 95 (1), 49-57. Available at: https://doi.org/10.1016/j.ress.2009.08.001. 